\title{
The first boundary value problem and the first eigenvalue problem for the elliptic equations degenerate on the boundary
}

\author{
By \\ Kazumasa Suzuki* \\ Introduction
}

The degenerate elliptic equations have been studied by many authors. Mikhlin [4] discussed those degenerate on a part of the boundary. However, he treated only the weak solutions of the problem. Il'in [2] and Oleinik [6] diszussed the degenerate elliptic equation

$$
\sum_{i, j=1}^{m} a_{i j} \frac{\partial^{2} u}{\partial x_{i} \partial x_{j}}+\sum_{i=1}^{m} b_{i} \frac{\partial u}{\partial x_{i}}+c u=f .
$$

They proved the uniqueness and existence theorems for the genuine solutions of the boundary value problem. They imposed some conditions on the equation, especially they required essentially that the equation is reduced to the case when $c \leqq c_{0}<0$ in the domain.

We treat the elliptic equation in the domain

$$
\sum_{i, j=1}^{m} \frac{\partial}{\partial x_{j}}\left(p_{i j} \frac{\partial u}{\partial x_{i}}\right)-q u=f
$$

which may be degenerate on the entire boundary. We prove, by the variational method, the uniqueness and existence theorems for the genuine solutions of the first boundary value problem and the existence theorem for the genuine solutions of the first eigenvalue problem. We do not assume that $q \geqq q_{0}>0$ in the domain. However, in order that Ladyzhenskaya-Ural'tseva's estimation for the solutions be applicable, we have to impose certain restrictions on the "order of de-

Received October 12, 1967.

Communicated by M. Hukuhara.

*Gakushûin High School. 
generacy".

In $\$ 1$ some notations and terminologies are introduced. We develop the main results in $\$ 2-\$ 4$; especially the boundary problems and the eigenvalue problems are stated in $\$ 3$ and in $\$ 4$ respectively. Section 5 is devoted to the lemmas used in $\$ 2-\$ 4$. In $\$ 6$ we give some sufficient conditions for the validity of the assumptions made in the main theorems. Finally we arrange, in $\$ 7$, some examples to which our theorems are applicable.

The author wishes to express his sincere thanks to Prof. M. Hukuhara for his helpful suggestions and constant encouragement.

\section{$\S 1$. Notations and terminologies}

Let $\Omega$ be a bounded domain in the $m$-dimensional Euclidean space, and $\partial \Omega$ its boundary.

By $C_{l, \alpha}(\bar{\Omega})(0<\alpha<1)$ we denote the space of functions $u$, the $l$-th order derivatives of which are Hölder continuous on $\Omega$ and for which

$$
\|u\|_{c_{l, \alpha}(\bar{\rho})} \equiv \sum_{k=0}^{l} \sum_{|\boldsymbol{k}|=k} \max _{\bar{\rho}}\left|D^{\boldsymbol{k}} u\right|+H_{l, \alpha, e}<\infty,
$$

where

$$
D^{\boldsymbol{k}} u \equiv \frac{\partial^{k_{1}+\cdots+k_{m}}}{\partial x_{1}^{k_{1} \cdots \partial x_{m}^{k_{m}}}},|\boldsymbol{k}| \equiv k_{1}+\cdots+k_{m}
$$

and

$$
H_{l, \alpha, \Omega} \equiv \max _{\mid \boldsymbol{k}^{\prime}=l} \sup _{x, x^{\prime} \in \Omega}\left|D^{k} u(x)-D^{k} u\left(x^{\prime}\right)\right| /\left|x-x^{\prime}\right|^{\alpha} .
$$

Also $C_{l, 0} \overline{(\Omega)}$ will denote the space of functions, the $l$-th order derivatives of which are continuous on $\bar{\Omega}$.

By $C_{l, \alpha}(\Omega)$ we denote the space of functions which belongs to $C_{l, \alpha}\left(\overline{\Omega^{\prime}}\right)$ for any domain $\Omega^{\prime}$ strictly contained in $\Omega$.

If for any $x_{0} \in \partial \Omega$, there exist a neighbourhood $U_{x_{0}}=\left\{x|| x-x_{0} \mid<\sigma_{x_{0}}\right\}$ and the local coordinates $y_{1}(x), \cdots, y_{m}(x) \in C_{l, \alpha}\left(\bar{U}_{x_{0}}\right)(0 \leqq \alpha<1)$ such that

$$
U_{x_{0}} \cap \bar{\Omega}=\left\{x \mid y_{m}(x) \geqq y_{m}\left(x_{0}\right)=0\right\} \cap U_{x_{0}}
$$

and 


$$
\left|\frac{D\left(y_{1}, \cdots, y_{m}\right)}{D\left(x_{1}, \cdots, x_{m}\right)}\right| \neq 0 \text { in } \bar{U}_{x_{0}}
$$

then we say that $\partial \Omega$ belongs to $C_{l, \alpha}$.

Let $\Omega_{1}, \cdots, \Omega_{n}, \cdots$ be the sequence of domains such that each member $\Omega_{n}$ is strictly contained in the next $\Omega_{n+1}$, and the boundary $\partial \Omega_{n}$ belongs to $C_{l, \alpha}$. If $\Omega=\bigcup_{n=1}^{\infty} \Omega_{n}$, then we say that $\partial \Omega$ belongs to $\bar{C}_{l, \alpha}$ and we call $\left\{\Omega_{n}\right\}$ an approximating sequence of domains for $\Omega(\partial \Omega \in$ $\bar{C}_{l, \alpha}$ ). Hereafter we assume $\partial \Omega$ belongs to $\bar{C}_{l, \alpha}$.

By $L_{p}(\Omega)$ we denote the space of functions $u$ which are measurable in $\Omega$ and for which

$$
\|u\|_{L_{\phi}(\rho)} \equiv\left[\int_{0}|u|^{p} d V\right]^{1 / p}<\infty .
$$

Let $\dot{C}_{\infty}(\Omega)$ be the set of functions which are infinitely differentiable in $\Omega$ and with supports strictly contained in $\Omega$.

When we have

$$
\int_{e} u \frac{\partial \zeta}{\partial x_{i}} d V=-\int_{0} w_{i} \zeta d V
$$

for the function $u$ defined in $\Omega$ and for every function $\zeta \in \dot{C}_{\infty}(\Omega)$, we call $w_{i}$ the generalized derivative of $u$ with respect to $x_{i}$ and denote it by $\frac{\partial u}{\partial x_{i}}$.

By $W_{p}^{(1)}(\Omega)$ we denote the space of functions $u$ measurable in $\Omega$ and having the first order generalized derivatives also measurable in $\Omega$, and for which

$$
\|u\| W_{p}^{(1)}(\Omega) \equiv\left[\int_{0}\left\{\sum_{i=1}^{m}\left(\frac{\partial u}{\partial x_{i}}\right)^{p}+u^{p} d\right\} V\right]^{1 / p}<\infty .
$$

By $\stackrel{\circ}{W}_{p}^{(1)}(\Omega)$ we denote the closure of $\dot{C}_{\infty}(\Omega)$ in $W_{p}^{(1)}(\Omega)$. For $\varphi \in C_{1,0}(\bar{\Omega})$ we shall denote by $\stackrel{\varphi}{W}_{p}^{(1)}(\Omega)$ the subset of $W_{p}^{(1)}(\Omega)$ defined by

$$
\stackrel{\rho}{W}_{p}^{(1)}(\Omega) \equiv\left\{u \mid u-\varphi \in \stackrel{\circ}{W}_{p}^{(1)}(\Omega)\right\} .
$$

For $u \in \stackrel{\phi}{W}_{p}^{(1)}(\Omega)$ and a real number $K$, we define

$$
u^{(K)} \equiv \max \{u-K, 0\}
$$


and

$$
\Omega^{(K)} \equiv\{x \in \Omega \mid u(x)>K\}
$$

For $p_{: j}(i, j=1, \cdots, m), q, \rho \in C_{0,0}(\bar{\Omega})$ and $\imath, v \in W_{2}^{(1)}(\Omega)$

we set

$$
\begin{aligned}
& G_{o}[u, v] \equiv \int_{o}\left\{\sum_{i=1}^{m} p_{i j} \frac{\partial u}{\partial x_{i}} \frac{\partial v}{\partial x_{j}}+q u v\right\} d V, \\
& D_{o}[u, v] \equiv \int_{o} \sum_{i, j=1}^{m} p_{i j} \frac{\partial u}{\partial x_{i}} \frac{\partial v}{\partial x_{j}} d V, \\
& H_{o, \rho}[u, v] \equiv \int_{o} \rho u v d V, \\
& G_{\odot}[u] \equiv G_{o}[u, u], D_{o}[u] \equiv D_{o}[u, u]
\end{aligned}
$$

and

$$
H_{\varrho, \rho}[u] \equiv H_{\varrho, \rho}[u, u] \text {. }
$$

Now we shall consider the boundary value problem

(1) $L[u] \equiv \sum_{i, j=1}^{m} \frac{\partial}{\partial x_{j}}\left(p_{i j} \frac{\partial u}{\partial x_{i}}\right)-q u=f \quad$ in $\Omega$,

(2) $u=\varphi \quad$ on $\partial \Omega$,

where $p_{i j}=p_{j i}, q, f \in C_{0,0}(\bar{\Omega})$ and $\varphi \in C_{1,0}(\bar{\Omega})$. We say that $u$ is a weak solution of (1)-(2) if

(i) $G_{\odot}[u, \zeta]+H_{o, 1}[f, \zeta]=0 \quad$ for every $\zeta \in \stackrel{\circ}{W}_{2}^{(1)}(\Omega)$, and

(ii) $D_{0}[u]<\infty$, and there exists $\left\{u_{h} \in \stackrel{\Phi}{W_{2}^{(1)}}(\Omega)\right\}$ such that $D_{o}\left[u-u_{h}\right] \rightarrow 0$ and $\left\|u-u_{h}\right\|_{L_{2}(\rho)} \rightarrow 0$ as $h \rightarrow \infty$.

If $u \in C_{2,0}(\Omega) \cap C_{0,0}(\bar{\Omega})$ satisfies $(1)-(2)$ and $D_{o}[u]<\infty$, then we call $u$ a solution of (1)-(2) (in this case $p_{i j} \in C_{1,0}(\Omega) \cap C_{0,0}(\bar{\Omega})$ ).

For the operator $L$ and a boundary point $x_{0} \in \partial \Omega$, we define a strong barrier function $v(x)$ as such that, for some $\sigma>0$,

(i) $v(x) \in C_{0,0}\left(\overline{\omega\left(x_{0}, \sigma\right)}\right)$

(ii) $v(x)=0$ at $x_{0}$

(iii) $v(x)>0$ in $\overline{\omega\left(x_{0}, \sigma\right)}-\left\{x_{0}\right\}$ 
(iv) $L[v]<0$ in $\omega\left(x_{0}, \sigma\right)$,

where $\omega\left(x_{0}, \sigma\right)=\left\{x \in \Omega|| x-x_{0} \mid<\sigma\right\}$.

To abbreviate the main theorems, we define Properties (A) and (B) of the domain and the operator $L$, as follows:

Property (A). For any boundary point $x_{0}$ there exists some $\sigma>0$, such that $\omega\left(x_{0}, \sigma\right) \equiv\left\{x \in \Omega|| x-x_{0} \mid<\sigma\right\}$ satisfies the following condition.

If $v \in C_{2,0}(\Omega) \cap C_{0,0}(\bar{\Omega})$ satisfies

$$
\begin{array}{ll}
L[v] \leqq \frac{\min }{\omega\left(x_{0}, \sigma\right)} f & \text { in } \omega\left(x_{0}, \sigma\right) \\
v \geqq \max _{\partial \omega\left(x_{0}, \rho\right)} \varphi & \text { on } \partial \omega\left(x_{0}, \sigma\right)
\end{array}
$$

resp.

$$
\begin{array}{ll}
L[v] \geqq \max _{\omega\left(x_{0}, \sigma\right)} f & \text { in } \omega\left(x_{0}, \sigma\right) \\
v \leqq \min _{\partial \omega\left(x_{0}, \sigma\right)} \varphi & \text { on } \partial \omega\left(x_{0}, \sigma\right),
\end{array}
$$

then for any solution $u$ of (1)-(2), we have

$$
u \leqq v \quad \text { in } \overline{\omega\left(x_{0}, \sigma\right)}
$$

resp.

$$
u \geqq v \quad \text { in } \overline{\omega\left(x_{0}, \sigma\right)} .
$$

Property (B). For any boundary point there is a strong barrier function.

\section{§2. Weak solutions}

In this section we consider the boundary value problem for the degenerate elliptic equation (1). In the first place we show that any solution of (1)-(2) is a weak solution of (1)-(2) (Theorem 1), and in the next place we give an a priori estimation for the weak solutions of Ladyzhenskaya-Ural'tseva's type (Theorem 2).

Theorem 1. If $u$ is a solution of

(1) $L[u] \equiv \sum_{i, j=1}^{m} \frac{\partial}{\partial x_{j}}\left(p_{i j} \frac{\partial u}{\partial x_{i}}\right)-q u=f \quad$ in $\Omega$,

(2) $u=\varphi \quad$ on $\partial \Omega$, 
then $u$ is a weak solution of (1)-(2), where

$$
\begin{aligned}
& p_{i j}=p_{j i} \in C_{1,0}(\Omega) \cap C_{0,0}(\bar{\Omega}) ; q, f \in C_{0,0}(\bar{\Omega}) ; \\
& \varphi \in C_{1,0}(\bar{\Omega}) ; \quad \partial \Omega \in \bar{C}_{1,0}
\end{aligned}
$$

and

$$
\sum_{i, j=1}^{m} p_{i j} \xi_{i} \xi_{j} \geqq 0\left(\left(\xi_{1}, \cdots, \xi_{m}\right) \text { is any real vector }\right) \text {. }
$$

Proof. For any $\xi \in \stackrel{\circ}{W}_{2}^{(1)}(\Omega)$ we take a sequence $\left\{\zeta_{h} \in \dot{C}_{\infty}(\Omega)\right\}$ such that $\zeta_{h} \rightarrow \zeta$ in $W_{2}^{(1)}(\Omega)$ as $h \rightarrow \infty$.

Since $u \in C_{2,0}(\Omega)$ satisfies (1), we have

$$
G_{o}\left[u, \zeta_{h}\right]+H_{o, 1}\left[f, \zeta_{h}\right]=0 .
$$

Therefore, by Lemma 1 and in a similar way we have

$$
\begin{aligned}
& G_{o}[u, \zeta]+H_{o, 1}[f, \zeta] \\
& =\lim _{h \rightarrow \infty}\left(G_{o}\left[u, \zeta_{h}\right]+H_{o, 1}\left[f, \zeta_{h}\right]\right)=0 .
\end{aligned}
$$

Moreover we can show that there exists a sequence $\left\{u_{h} \in W_{2}^{(1)}(\Omega)\right\}$ such that $D_{Q}\left[u-u_{h}\right] \rightarrow 0$ and $\left\|u-u_{h}\right\|_{L_{2}(\rho)} \rightarrow 0$, as follows.

Since $D_{o}[u]$ is finite, we get

$$
D_{s}[u-\varphi] \leqq\left(\sqrt{D_{0}[u]}+\sqrt{D_{0}[\varphi]}\right)^{2}<\infty .
$$

Choose an approximating sequence $\left\{\Omega_{h}\right\}$ for $\Omega\left(\partial \Omega \in \bar{C}_{1,0}\right)$, and set

$$
u_{h} \equiv \begin{cases}u-K_{h} & \text { in } \Omega_{h}^{+} \equiv\left\{x \in \Omega_{h} \mid u(x)-\varphi(x)>K_{h}\right\} \\ u+K_{h} & \text { in } \Omega_{h}^{-} \equiv\left\{x \in \Omega_{h} \mid u(x)-\varphi(x)<-K_{h}\right\} \\ \varphi & \text { in } \Omega_{h}^{0} \quad\left\{x \in \Omega_{h}|| u(x)-\varphi(x) \mid \leqq K_{h}\right\} \\ \varphi & \text { in } \Omega-\Omega_{h}\end{cases}
$$

where $K_{h} \equiv \max _{\partial \varrho_{h}}|u-\varphi| \rightarrow 0$ as $h \rightarrow \infty$.

Then we have

$$
\begin{aligned}
& \left\|u-u_{h}\right\|_{L_{2}(\rho)}=\left\|u-u_{h}\right\|_{L_{2}\left(\rho-\Omega_{h}\right)}+\left\|K_{h}\right\|_{L_{2}(g h)}^{+} \\
& +\left\|-K_{h}\right\|_{L_{2}\left(\rho_{\bar{h}}^{-}\right)}+\|u-\varphi\|_{L_{2}(\rho h)}^{o} \\
& \rightarrow \lim _{h \rightarrow \infty}\|u-\varphi\|_{L(\Omega h)}
\end{aligned}
$$


and

$$
\begin{aligned}
& D_{o}\left[u-u_{i}\right] \\
& =D_{\Omega_{-}}[u-\varphi]+D_{\Omega_{h}^{+}}\left[K_{h}\right]+D_{o_{h}^{-}}\left[-K_{h}\right]+D_{o_{h}^{o}}^{o}[u-\varphi] \\
& \rightarrow \lim _{h \rightarrow \infty} D_{\Omega_{h}^{o}}^{o}[u-\varphi] .
\end{aligned}
$$

On the other hand

$$
\operatorname{mes}\left(\Omega_{h}^{0}-\{x \in \Omega \mid u(x)=\varphi(x)\}\right) \rightarrow 0,
$$

and therefore

$$
\begin{aligned}
& \lim _{h \rightarrow \infty}\|u-\varphi\|_{L_{2}(o h)}^{o}=0, \\
& \lim _{h \rightarrow \infty} D_{o h}^{o}[u-\varphi]=0 .
\end{aligned}
$$

Thus

$$
\left\|u-u_{h}\right\|_{L_{2}(\rho)} \rightarrow 0 \quad(h \rightarrow \infty)
$$

and

$$
D_{o}\left[u-u_{h}\right] \rightarrow 0 \quad(h \rightarrow \infty) .
$$

Finally we show that $u_{h} \in W_{2}^{(1)}(\Omega)$. Note that $u-\varphi \in C_{1,0}\left(\Omega_{h}\right)$, which implies that $u-\varphi \in \stackrel{u-\phi}{W} W_{2}^{(1)}\left(\Omega_{h}\right)$. Therefore, if we set

$$
\begin{aligned}
& v_{h}^{+} \equiv \begin{cases}\max \left\{u-\varphi-K_{h}, 0\right\} & \text { in } \Omega_{h} \\
0 & \text { in } \Omega-\Omega_{h}\end{cases} \\
& v_{h}^{-} \equiv \begin{cases}\max \left\{u-\varphi+K_{h}, 0\right\} & \text { in } \Omega_{h} \\
0 & \text { in } \Omega-\Omega_{h},\end{cases}
\end{aligned}
$$

then $v_{h}^{+}, v_{h}^{-} \in \stackrel{\circ}{W}_{2}^{(1)}(\Omega)$ (cf. Lemma 2). Thus

$$
u_{h}-\varphi=v_{h}^{+}+v_{h}^{-} \in \stackrel{\circ}{W_{2}^{(1)}}(\Omega) \text {, }
$$

i. e.,

$$
u_{h} \in \stackrel{\varphi}{W}(1)(\Omega)
$$

Theorem 2. Assume that $p_{i j}=p_{j i} q, f \in C_{0,0} \overline{(\Omega)}$ and $\varphi \in C_{1,0}(\bar{\Omega})$, and that $p_{i j}$ satisfies

$$
\sum_{i, j=1}^{m} p_{i j} \xi_{i} \xi_{j} \geqq p_{0}(x) \sum_{i=1}^{m} \xi_{i}^{2} \geqq 0
$$




$$
\left(\left(\xi_{1}, \cdots, \xi_{m}\right) \text { is any real vector }\right)
$$

where

when $m=1$ ).

$$
\frac{1}{p_{0}} \in L_{k /(1-k)}(\Omega)\left(\frac{m}{m+2}<k<1 \text { when } m \geqq 2 \text { or } \frac{1}{2}<k<1\right.
$$

If $u$ is a weak solution of (1)-(2) which satisfies $D_{\bullet}[u]<d$, then

$$
\underset{o}{\operatorname{vrai} \max }|u|<C \text {, }
$$

when $C$ depends on $m, k, d$ and $C^{\prime}\left(C^{\prime}\right.$ is any positive constant such that $\left\|\frac{1}{p_{0}}\right\|_{L^{L /(1-k)(\rho)},}, \max _{\bar{a}}|q|, \max _{\overline{\bar{a}}}|f|, \max _{\overline{\bar{a}}}|\varphi|, D_{\Omega}[\varphi]$, mes $\left.\Omega<C^{\prime}\right)$.

Proof. We begin with the case $m \geqq 2$. Since $u$ is a weak solution of (1)-(2), and $u^{(K)} \in \stackrel{\circ}{W}_{2}^{(1)}(\Omega)\left(K \geqq \max _{\partial \rho} \varphi, K \geqq 1\right)$, we have

$$
\left.\int_{e}(K) \sum_{i, j=1}^{m} p_{i j} \frac{\partial u}{\partial x_{i}} \frac{\partial u^{(K)}}{\partial x_{j}}+q u u^{(K)}+f u^{(K)}\right] d V=0 .
$$

Therefore, we have

$$
\begin{aligned}
& \int_{Q}(K) \sum_{i, j=1}^{m} p_{i j} \frac{\partial u}{\partial x_{i}} \frac{\partial u}{\partial x_{j}} d V \\
& \leqq \max _{\bar{a}}|q| \int_{e}(K) u(u-K) d V+\int_{e}(K)|f|(u-K) d V \\
& \leqq \max _{\overline{\bar{Q}}}|q|\left[-\frac{3}{2} \int_{\Omega}(K)(u-K)^{2} d V+\frac{K^{2}}{2} \operatorname{mes} \Omega^{(K)}\right] \\
& +\frac{1}{2} \int_{\Omega}(K)(u-K)^{2} d V+\frac{1}{2} \max _{=}|f|^{2} \operatorname{mes} \Omega^{(K)} \\
& \leqq 2 \max \left\{\max _{\bar{u}}|q|, \max _{\bar{u}}|f|^{2}, 1\right\}\left[\int_{\Omega}(K)(u-K)^{2} d V+K^{2} \operatorname{mes} \Omega^{(K)}\right] .
\end{aligned}
$$

Now by Corollary 1 to Lemma 4 and Corollary 1 to Lemma 8, we get

$$
\begin{aligned}
& \|\nabla u\|_{L_{2 k}\left(Q^{(K)}\right)}^{2} \leqq 2\left\|\frac{1}{p_{0}}\right\|_{L k /(1-k)(e)} \\
& \times \max \left\{\max _{\bar{a}}|q|, \max _{\bar{a}}|f|^{2}, 1\right\}\left[B_{m b}{ }^{\prime 2}\|\nabla u\|_{L_{2 k}(\theta)^{(X)}}^{2}+K^{2} \operatorname{mes} \Omega^{(K)}\right] .
\end{aligned}
$$

Whereas 


$$
\begin{aligned}
& \left(K^{2 k m /(m-2 k)} \operatorname{mes} \Omega^{(K)}\right)^{\{k(m+2)-m\} / k m} \\
& \leqq\left[\int_{0}(K) u^{2 k m /(m-2 k)} d V\right]^{\{k(m+9)-m\} / k m} \leqq\|u\|_{L_{2 k m /(m-2 k)}^{2(k(㇒)}}^{2(\Omega)+2)-m\} /(m-2 k)} \\
& \leqq\left[\|U-\varphi\|_{L_{2 k m /(m-2 k)(\rho)}}+\|\varphi\|_{L_{2 k n /(m-2 k)(\rho)}}\right]^{2[k(m+2)-m] /(m-2 k)} \text {, }
\end{aligned}
$$

and $u-\varphi \in \stackrel{\circ}{W}_{2}^{(1)}(\Omega)$, so that from Lemma 3 and Lemma 8 follows

$$
\begin{aligned}
& \left(K^{2 k m /(m-2 k)} \operatorname{mes} \Omega^{(K)}\right)^{\{k(m+2)-m\} / k m} \\
& \leqq\left\{\frac{k(m-1)}{m-2 k}\left(\left\|\frac{1}{p_{0}}\right\|_{L k / 11-k)(o)} D_{o}[u-\varphi]\right)^{1 / 2}\right. \\
& \left.+\|\varphi\|_{L 2 k m /(m-2 k)(\rho)}\right\}^{2[k(m+2)-m\} /(m-2 k)} \\
& \leqq\left\{\frac{k(m-1)}{m-2 k}\left(\left\|\frac{1}{p_{0}}\right\|_{L_{k / 1-k)(\Omega)}}\right)^{1 / 2}\left(\sqrt{D_{o}[u]}+\sqrt{D_{o}[\varphi]}\right)\right. \\
& \left.+\|\varphi\|_{L 2 k m /\langle m-2 k)(\rho)}\right\}^{2\{(m+2)-m\} /(m-2 k)},
\end{aligned}
$$

which implies that mes $\Omega^{(K)} \rightarrow 0$ as $K \rightarrow \infty$.

Thus if we choose $K_{0}\left(\geqq \max _{\partial \rho} \varphi, 1\right)$ so large that

$$
\begin{aligned}
2 \| \frac{1}{p_{0}} & \|_{L k /(1-k)(\rho)} \max \left\{\max _{\bar{\rho}}|q|, \max _{\bar{\rho}} \mid f !^{2}, 1\right\} B_{m k^{\prime}} \\
& \leqq 2\left\|\frac{1}{p_{0}}\right\|_{L k /(1-k)(\rho)} \max \left\{\max _{\bar{a}}|q|, \max _{\bar{\rho}}|f|^{2}, 1\right\} \\
& \times\left(\frac{k(m-1)}{m-2 k}\right)^{2}\left(\operatorname{mes} \Omega^{\left(K_{0}\right)}\right)^{\{(m+2) k-m\} / m k} \leqq \frac{1}{2},
\end{aligned}
$$

then for any $K \geqq K_{0}$ we have

$$
\|\nabla u\|_{L_{2 k(Q(K)}(K)}^{2} \leqq 4\left\|\frac{1}{p_{0}}\right\|_{L k /(1-k)(Q)} \times \max \left\{\max _{\bar{\rho}}|q|, \max _{\bar{\rho}}|f|^{2}, 1\right\} K^{2} \operatorname{mes} \Omega^{(K)} .
$$

Hence the assumption of Lemma 7 is satisfied.

Next we show that $\|u\|_{L_{1}\left(Q^{(K} K_{0}\right)}$ is bounded by a constant which does not depend on $u$.

Since

$$
\begin{gathered}
\|u\|_{L_{1}\left(\rho^{\left(K_{0}\right)}\right)} \leqq(\operatorname{mes} \Omega)^{1 / 2}\|u\|_{\left.L_{2}\left(\rho^{(K}\right)\right)} \leqq(\operatorname{mes} \Omega)^{1 / 2}\left\|u^{\left(K_{0}\right)}+K_{0}\right\|_{L_{2}\left(Q^{\left(K_{0}\right)}\right)} \\
\leqq(\operatorname{mes} \Omega)^{1 / 2}\left(\left\|u^{\left(K_{0}\right)}\right\|_{L_{2}\left(\rho\left(K_{0}\right)\right)}+K_{0}(\operatorname{mes} \Omega)^{1 / 2}\right),
\end{gathered}
$$

from Corollary 2 to Lemma 8 follows 


$$
\begin{aligned}
& \|u\|_{L_{1}\left(\mathcal{G}^{(}\left(K_{0}\right)\right)} \leqq(\operatorname{mes} \Omega)^{1 / 2} \\
& \times\left\{\frac{k(m-1)}{m-2 k}(\operatorname{mes} \Omega)^{\{(m+2) k-m\} / 2 m k} \sqrt{\left\|\frac{1}{p_{0}}\right\|_{L_{k /(1-k)(\Omega)}} D_{\left.D^{(K O}\right)}[u]}\right. \\
& \left.\quad+K_{0}(\operatorname{mes} \Omega)^{1 / 2}\right\} .
\end{aligned}
$$

Therefore by Lemma 7 vrai $\max u$ is bounded by a constant $C$ which depends on $m, k, d$ and $C^{\prime}$.

Since $-u$ is a weak solution of (1)-(2) in which $f$ and $\varphi$ are replaced by $-f$ and $-\varphi$ respectively, $\operatorname{vrai}_{\rho} \max (-u)$ is also bounded by $C$.

Thus we have

$$
\text { vrai } \max _{a}|u|<C \text {, }
$$

which completes the proof in the case $m \geqq 2$.

When $m=1$, by Lemmas 2,8 , and (5) in the proof of Lemma 4 , we set

$$
|u-\varphi| \leqq \frac{1}{2}(\operatorname{mes} \Omega)^{(2 k-1) / 2 k} \sqrt{\left\|\frac{1}{p_{0}}\right\|_{L k /(1-k)(\rho)} D_{o}[u]}
$$

Therefore

$$
|u| \leqq|u-\varphi|+|\varphi|<C
$$

\section{§3. Boundary value problems}

We treat the boundary value problem for the degenerate elliptic equation

$$
\sum_{i, j=1}^{m} \frac{\partial}{\partial x_{j}}\left(p_{i j} \frac{\partial u}{\partial x_{i}}\right)-q u=f \quad \text { in } \Omega
$$

with the boundary condition

$$
u=\varphi \quad \text { on } \partial \Omega .
$$

We give the uniqueness theorem for weak solutions (Theorem 3) and the existence theorem for genuine solutions(Theorem 4).

Theorem 3. Assume that $p_{i j}=p_{j i}, q, f \in C_{0,0}(\bar{\Omega}) ; \varphi \in C_{1,0}(\bar{\Omega})$ and $\partial \Omega \in \bar{C}_{1,0}$ and also that $p_{i j}$ and $q$ satisfy

$$
\sum_{i, j=1}^{m} p_{i j} \xi_{i} \xi_{j} \geqq p_{0}(x) \sum_{i=1}^{m} \xi_{i}^{2} \geqq 0
$$


$\left(\left(\xi_{1}, \cdots, \xi_{m}\right)\right.$ is any real vector $)$

$\frac{1}{p_{0}} \in L_{k / 1-k)}(\Omega)$ (for some $k$ with $\frac{m}{m+2}<k<1$ when $m \geqq 2$,

or with $\frac{1}{2}<k<1$ when $m=1$ )

and

$q \geqq 0$

in $\Omega$.

Then the weak solution of (1)-(2) is unique.

Proof. Let $u_{1}$ and $u_{2}$ be two weak solutions of (1)-(2). Then we have

$$
G_{o}\left[u_{1}, \zeta\right]+H_{o, 1}[f, \zeta]=0
$$

and

$$
G_{\odot}\left[u_{2}, \zeta\right]+H_{o, 1}[f, \zeta]=0
$$

for every $\zeta \in \stackrel{\circ}{W}_{2}^{(1)}(\Omega)$. Therefore we get

$$
G_{o}\left[u_{1}-u_{2}, \zeta\right]=0 .
$$

By the definition of a weak solution, there exist two sequences $\left\{u_{1 h} \in \stackrel{\Phi}{W}_{2}^{(1)}(\Omega)\right\}$ and $\left\{u_{2 h} \in \stackrel{\Phi}{W}_{2}^{(1)}(\Omega)\right\}$ such that

$$
D_{e}\left[u_{1}-u_{1 k}\right] \rightarrow 0,\left\|u_{1}-u_{1 h}\right\|_{L_{2}(e)} \rightarrow 0
$$

and

$$
D_{\varrho}\left[u_{2}-u_{2 h}\right] \rightarrow 0,\left\|u_{2}-u_{2 h}\right\|_{L_{2}(\rho)} \rightarrow 0 \quad \text { as } h \rightarrow \infty \text {. }
$$

Now set $\zeta=u_{1 \hbar}-u_{2 \hbar}$. Then we have

$$
G_{\varrho}\left\lceil u_{1}-u_{2}, u_{1 i}-u_{2 i}\right\rceil=0 .
$$

From Lemma 1 follows

$$
G_{o}\left[u_{1}-u_{2}\right]=0
$$

and therefore, because of $q \geqq 0$,

$$
D_{s}\left[u_{1}-u_{2}\right]=0 \text {. }
$$

Thus by Corollary 2 to Lemma 8 we have

$$
\left\|u_{1}-u_{2}\right\|_{L_{2}(\theta)}=\lim _{h \rightarrow \infty}\left\|u_{1 h}-u_{2 h}\right\|_{L_{2}(\theta)}
$$




$$
\leqq \lim _{h \rightarrow \infty} B_{m k}^{\prime} \sqrt{\left\|\frac{1}{p_{0}}\right\|_{L k /(1-k)(o)} D_{o}\left[u_{1 k}-u_{2 \hbar}\right]}=0,
$$

i. e., $u_{1}=u_{2}$ almost everywhere in $\Omega$.

Corollary. Assume that $p_{i j}=p_{j i} \in C_{1,0}(\Omega) \cap C_{0,0}(\bar{\Omega}) ; q, f \in C_{0,0}(\bar{\Omega})$; $\varphi \in C_{1,0}(\bar{\Omega})$ and $\partial \Omega \in \bar{C}_{1,0}$ and also that $p_{i j}$ and $q$ satisfy

$$
\begin{aligned}
& \sum_{i, j=1}^{m} p_{i j} \xi_{i} \xi_{j} \geqq p_{0}(x) \sum_{i=1}^{m} \xi_{i}^{2} \geqq 0 \\
& \quad\left(\left(\xi_{1}, \cdots, \xi_{m}\right)\right. \text { is any real vector) } \\
& \frac{1}{p_{0}} \in L_{k /(1-k)}(\Omega) \\
& \text { (for some } k \text { with } \frac{m}{m+2}<k<1 \text { when } m \geqq 2 \text {, or with }
\end{aligned}
$$

$\frac{1}{2}<k<1$ when $m=1$ )

and

$$
q \geqq 0
$$

in $\Omega$.

Then the solution of (1)-(2) is unique.

This corollary is clear by Theorem 1 and Theorem 3.

Theorem 4. Assume that the bounded domain $\Omega$ in m-dimensional Euclidean space and the coefficients of the problem (1)(2) satisfy the following conditions:

$$
\begin{aligned}
& p_{i j}=p_{j i}, q, f \in C_{0, \alpha}(\Omega)_{\cap} C_{0,0}(\bar{\Omega}), \\
& \frac{\partial p_{i j}}{\partial x_{j}} \in C_{0, \alpha}(\Omega)(i, j=1, \cdots, m), \varphi \in C_{2, \alpha}(\Omega)_{\cap} C_{1,0}(\bar{\Omega}), \\
& \partial \Omega \in \bar{C}_{2, \alpha}, \\
& \sum_{i, j=1}^{m} p_{i j} \xi_{i} \xi_{j} \geqq p_{0}(x) \sum_{i=1}^{m} \xi_{i}^{2} \geqq 0 \quad\left(\left(\xi_{1}, \cdots, \xi_{m}\right)\right. \text { is any real vector), } \\
& \frac{1}{p_{0}} \in L_{k /(1-k)}(\Omega) \\
& \text { (for some } k \text { with } \frac{m}{m+2}<k<1 \text { when } m \geqq 2 \text {, or with }
\end{aligned}
$$


$\frac{1}{2}<k<1$ when $m=1$ ),

$$
\begin{array}{ll}
p_{0}(x)>0 & \text { in } \Omega, \\
q \geqq 0 & \text { in } \bar{\Omega},
\end{array}
$$

(ii) the domain and the operator have properties $(A)$ and $(B)$. Then there exists a unique solution $u_{0}$ of (1)-(2). Moreover we have

$$
\begin{aligned}
& G_{o}\left[u_{0}\right]+2 H_{o, 1}\left[f, u_{0}\right] \\
& =\inf _{\substack{q(1) \\
u \in W^{\prime}(o)}}\left(G_{\diamond}[u]+2 H_{o, 1}[f, u]\right) .
\end{aligned}
$$

Proof. By Lemma 9, there exist $d$ and $d_{n}$ such that

$$
\inf _{u \in \in_{W 2}^{\phi(1)}}\left(G_{\varrho}[u]+2 H_{e, 1}[f, u]\right)=d
$$

and

$$
\inf _{\substack{\boldsymbol{\rho}(1) \\ u \in \in_{W 2}\left(\rho_{)}\right)}}\left(G_{\rho_{n}}[u]+2 H_{\varrho_{n}, 1}[f, u]\right)=d_{n}
$$

where $\left\{\Omega_{n}\right\}$ is an approximating sequence for $\Omega\left(\partial \Omega \in \bar{C}_{2, \alpha}\right)$. Clearly

$$
d_{1} \geqq \cdots \geqq d_{n} \geqq \cdots \geqq d .
$$

Moreover we can show that $\lim _{n \rightarrow \infty} d_{n}=d$.

In fact, there exists $\left\{u_{h} \in \stackrel{\Phi}{W}_{2}^{(1)}(\Omega)\right\}$ such that

$$
\lim _{h \rightarrow \infty}\left(G_{o}\left[u_{h}\right]+2 H_{o, 1}\left[f, u_{h}\right]\right)=d
$$

and there exists $\left\{u_{k}^{\prime} \mid u_{k}^{\prime}-\varphi \in \dot{C}_{\infty}(\Omega)\right\}$ such that

$$
\left|G_{\diamond}\left[u_{h}\right]-G_{\diamond}\left[u_{h}^{\prime}\right]\right|<\frac{1}{h}
$$

and

$$
\left|H_{e, 1}\left[f, u_{h}\right]-H_{e, 1}\left[f, u_{h}^{\prime}\right]\right|<\frac{1}{h} .
$$

By choosing $n$ so large that $u_{h}^{\prime} \in \stackrel{\varphi}{W}_{2}^{(1)}\left(\Omega_{n}\right)$, we have

$$
\begin{aligned}
d & =\lim _{h \rightarrow \infty}\left(G_{\varrho}\left[u_{h}\right]+2 H_{o, 1}\left[f, u_{h}\right]\right) \\
& =\lim _{h \rightarrow \infty}\left(G_{\rho}\left[u_{h}^{\prime}\right]+2 H_{o, 1}\left[f, u_{h}^{\prime}\right]\right)
\end{aligned}
$$




$$
\begin{aligned}
& \geqq \lim _{h \rightarrow \infty} \inf _{u \in \in_{W 2}^{\phi(1)}\left(\varrho_{n}\right)}\left(G_{\rho_{n}}[u]+2 H_{\rho_{n}, 1}[f, u]\right) \\
& =\lim _{h \rightarrow \infty} d_{h(h) .} .
\end{aligned}
$$

Hence

$$
\lim _{u \rightarrow \infty} d_{n}=d .
$$

Whereas it is certain that for each $n$ there exists a solution $u_{n} \in C_{2, \alpha}\left(\Omega_{n}\right)$ of (1)-(2) for $\Omega_{n}$, which satisfies

$$
G_{Q_{n}}\left[u_{n}\right]+2 H_{\rho_{n, 1}}\left[f, u_{n}\right] \underset{\substack{u \in \in_{W 2}^{\Phi(1)} \\=\inf }}{ }\left(G_{o_{n}}[u]+2 H_{o_{n}, 1}[f, u]\right) .^{1)}
$$

Therefore we have

$$
d_{n}=G_{Q_{n}}\left[u_{n}\right]+2 H_{{\vartheta_{n}, 1}_{1}}\left[f, u_{n}\right]
$$

which are bounded above by $d_{1}$. By Lemma $10 D_{o_{n}}\left[u_{n}\right]$ is bounded and therefore, if we define $u_{n}=\varphi$ in $\Omega-\Omega_{n}$,

$$
D_{o}\left[u_{n}\right]=D_{o_{n}}\left[u_{n}\right]+D_{o_{-} o_{n}}[\varphi]
$$

is also bounded. Since by Theorem $1 u_{n}$ is a weak solution of (1)(2) for $\Omega_{n}$, from Theorem 2 follows

$$
\max _{\overline{\boldsymbol{s}}_{n}}\left|u_{n}\right|<C
$$

where $C$ depends only on $m, k, d_{1},\left\|\frac{1}{p_{0}}\right\|_{L k /(1-k)(\theta)}, \max _{\overline{0}}|q|$, $\max _{\bar{a}}|f|, \max _{\bar{\Omega}}|\varphi|, D_{o}[\varphi]$ and mes $\Omega$. Hence

$$
\max _{\vec{a}}\left|u_{n}\right|<C^{\prime} \text {, }
$$

where $C^{\prime}$ depends on the same quantities as $C$.

By Lemma $12 u_{n^{\prime}}\left(n^{\prime}>n\right)$ satisfies

$$
\begin{aligned}
& \left\|u_{n^{\prime}}\right\|_{c_{2, \alpha}}\left(\bar{\Omega}_{n}\right) \leqq C^{\prime \prime}\left[\left\|L u_{n^{\prime}}\right\| C_{0, \alpha}\left(\bar{\Omega}_{n+1}\right)+\max _{\bar{\Omega}_{n+1}}\left|u_{n^{\prime}}\right|\right] \\
& <C^{\prime \prime}\left[\|f\|_{c_{0, \alpha}(\bar{\Omega})}+C^{\prime}\right]<C^{\prime \prime \prime}
\end{aligned}
$$

where $C^{\prime \prime \prime}$ depends on $\alpha,\left\|\frac{\partial p_{i j}}{\partial x_{j}}\right\|_{c_{0, \alpha}\left(\bar{\Omega}_{n+1}\right)},\|q\|_{C_{0, \alpha}\left(\bar{\Omega}_{n+1}\right)}, \min _{\bar{\rho}_{n+1}} p_{0}$, the diame-

1) This is shown in the same way as in Suzuki [9], where the solution of the first eigenvalue problem are derived-by the variational method. 
ter of $\Omega_{n+1}$, the distance between $\Omega_{n}$ and $\partial \Omega_{n+1},\|f\|_{c_{0, \alpha}(\bar{\Omega})}$ and $C^{\prime}$.

Therefore we can choose the subsequence of $\left\{u_{n}\right\}$ such that

$$
\begin{aligned}
& u_{11}, u_{12}, \cdots, u_{1 n}, \cdots \rightarrow u_{0} \text { in } C_{2,0}\left(\bar{\Omega}_{1}\right) \\
& u_{21}, u_{22}, \cdots, u_{2 n}, \cdots \rightarrow u_{0} \text { in } C_{2,0}\left(\bar{\Omega}_{2}\right) \\
& \ldots \\
& u_{n 1}, u_{n 2}, \cdots, u_{n n}, \cdots \rightarrow u_{0} \text { in } C_{2,0}\left(\bar{\Omega}_{n}\right) \\
& \cdots,
\end{aligned}
$$

where the upper subsequence contains the lower subsequence and $u_{0} \in C_{2, \alpha}(\Omega),{ }^{2)}$ the absolute value of which is bounded by $C^{\prime \prime \prime}$. Obviously $u_{0}$ satisfies $(1)$ in $\Omega$, and $D D_{2}\left[u_{0}\right]=\lim _{n \rightarrow \infty} D_{o_{n}}\left[u_{0}\right]=\lim _{n \rightarrow \infty} \lim _{n^{\prime} \rightarrow \infty} D_{s_{n}}\left[u_{n^{\prime} n^{\prime}}\right]$ is finite.

Let us show that $u_{0}$ satisfies (2) at the boundary.

By Property (B), there exists a strong barrier function $v(x)$ for any boundary point $x_{0}$. Set

$$
\psi_{1}(x)=\varphi\left(x_{0}\right)+\varepsilon+k_{1} v(x)
$$

and

$$
\psi_{2}(x)=\varphi\left(x_{0}\right)-\varepsilon-k_{2} v(x) .
$$

Then we have

$$
\psi_{1}(x) \geqq \psi_{2}(x) .
$$

In the first place we choose $\omega\left(x_{0}, \sigma\right)$, which appears in Properties (A) and (B), so small that

$$
\psi_{1}(x) \geqq \max _{\partial \Omega \cap \partial \omega\left(\lambda_{0}, \sigma\right)} u_{n}
$$

and

$$
\psi r_{2}(x) \leqq \min _{\partial \rho \cap \partial \omega\left(x_{0}, \sigma\right)} u_{n}
$$

on $\partial \Omega \cap \partial \omega\left(x_{0}, \sigma\right)$. In the next place we choose $k_{1}, k_{2}$ so large that

$$
L\left[\psi_{1}(x)\right] \leqq \frac{\min }{\omega\left(x_{0}, \sigma\right)} f
$$

2) If $\left\|u_{n}\right\| \|_{2}, \alpha(\bar{\Omega})$ are bounded and $u_{r 0} \rightarrow u_{0}$ in $C_{2,0}(\bar{\Omega})$, then $u_{0} \in C_{2, \alpha}(\bar{\Omega})$ (cf. Suzuki [9] p. 68, Theorem 9). 


$$
L\left[\psi_{2}(x)\right] \geqq \frac{\max }{\omega\left(x_{0}, \sigma\right)} f
$$

in $\omega\left(x_{0}, \sigma\right)$, and

$$
\begin{aligned}
& \psi_{1}(x) \geqq \max _{\partial \omega\left(x_{0}, \sigma\right)} u_{n}, \\
& \psi_{2}(x) \leqq \min _{\partial \omega\left(x_{0}, \sigma\right)} u_{n}
\end{aligned}
$$

on $\partial \omega\left(x_{0}, \sigma\right)$. Then by Property (A), we have

$$
\psi r_{2}(x) \leqq u_{n}(x) \leqq \psi_{1}(x) \text { in } \omega\left(x_{0}, \sigma\right) .
$$

Therefore, letting $n \rightarrow \infty$, we have

$$
\psi_{2}(x) \leqq u_{0}(x) \leqq \psi_{1}(x) \text { in } \omega\left(x_{0}, \sigma\right) .
$$

Hence, letting $x \rightarrow x_{0}$ and letting $\varepsilon \rightarrow 0$, we obtain

$$
\varphi\left(x_{0}\right)=\varlimsup_{x \rightarrow x_{0}} u_{0}(x) \leqq \varlimsup_{x \rightarrow x_{0}} u_{0}(x)=\varphi\left(x_{0}\right) .
$$

Therefore

$$
\lim _{x \rightarrow x_{0}} u_{0}(x)=\varphi\left(x_{0}\right) .
$$

Thus we have shown the existence of a solution of the problem (1)(2). Because of Theorem 3 , this solution is unique.

Finally we shall show that we have

$$
G_{o}\left[u_{0}\right]+2 H_{Q, 1}\left[f, u_{0}\right]=d .
$$

Since $u_{0}$ is a solution of the problem (1)-(2), by Theorem $1 u_{0}$ is also a weak solution of the problem (1)-(2), i. e., there exists a sequence $\left\{u_{h} \in \stackrel{\Phi}{W}_{2}^{(1)}(\Omega)\right\}$ such that $D_{s}\left[u_{0}-u_{h}\right] \rightarrow 0,\left\|u_{0}-u_{h}\right\|_{L_{2}(\theta)} \rightarrow 0$ as $h \rightarrow \infty$, and for any $\zeta \in \stackrel{\circ}{W}_{2}^{(1)}(\Omega)$ we have

$$
G_{o}\left[u_{0}, \zeta\right]+H_{o, 1}[f, \zeta]=0 .
$$

Therefore, from Lemma 1 it follows that

$$
\begin{aligned}
G_{o}\left[u_{0}\right]+2 H_{o, 1}\left[f, u_{0}\right] \\
\quad=\lim _{h \rightarrow \infty}\left(G_{o}\left[u_{h}\right]+2 H_{o, 1}\left[f, u_{h}\right]\right) \\
\geqq \lim _{h \rightarrow \infty} d=d .
\end{aligned}
$$

For any $u \in \stackrel{\varphi}{W}{ }_{2}^{(1)}(\Omega)$, put 


$$
u=u_{0}+\zeta, u=u_{h}+\zeta_{h} .
$$

Clearly $\xi_{h} \in \stackrel{\circ}{W}_{2}^{(1)}(\Omega)$ and

$$
D_{o}\left[\zeta-\zeta_{h}\right] \rightarrow 0,\left\|\zeta-\zeta_{h}\right\|_{L_{2}(\rho)} \rightarrow 0 \text { as } h \rightarrow \infty .
$$

Therefore

$$
\begin{aligned}
& \left(G_{\circ}[u]+2 H_{\varrho, 1}[f, u]\right)-\left(G_{\odot}\left[u_{h}\right]+2 H_{\rho_{1} 1}\left[f, u_{h}\right]\right) \\
& =G_{o}\left[\zeta_{h}\right]+\left(G_{\rho}\left[u_{h}, \zeta_{h}\right]+H_{o, 1}\left[f, \zeta_{h}\right]\right) \\
& \geqq G_{\rho}\left[u_{h}, \zeta_{h}\right]+H_{Q, 1}\left[f, \zeta_{h}\right] \text {. }
\end{aligned}
$$

By Lemma 1, letting $h \rightarrow \infty$, we have

$$
\begin{aligned}
\left(G_{o}\right. & {\left.[u]+2 H_{o, 1}[f, u]\right)-\left(G_{o}\left[u_{0}\right]+2 H_{o, 1}\left[f, u_{0}\right]\right) } \\
& \left.\left.\geqq G_{\diamond}\left[u_{0}, \zeta\right]+H_{o, 1}\right] f, \zeta\right] \\
& =\lim _{h \rightarrow \infty}\left(G_{o}\left[u_{0}, \zeta_{h}\right]+H_{o, 1}\left[f, \zeta_{h}\right]\right)=0 .
\end{aligned}
$$

Thus we have

$$
G_{o}\left[u_{0}\right]+2 H_{o, 1}\left[f, u_{0}\right\rfloor \leqq d .
$$

Hence we have

$$
G_{\diamond}\left[u_{0}\right]+2 H_{\iota, 1}\left[f, u_{0}\right]=d .
$$

\section{§4. Eigenvalue problems}

We consider the eigenvalue problem for the degenerate elliptic equation

$$
\sum_{i, j=1}^{n} \frac{\partial}{\partial x_{j}}\left(p_{i j} \frac{\partial u}{\partial x_{i}}\right)-q u+\lambda \rho u=0 \quad \text { in } \Omega,
$$

with the boundary condition

$$
u=0 \quad \text { on } \partial \Omega \text {. }
$$

We arrange the fundamental properties of the eigenfunctions and eigenvalues (Proposition 1-2). Next we prove the discreteness of the spectrum (Theorem 5), and finally we give the solution of the problem for (3)-(4) which shows that the number of eigenvalues is indeed countable (Theorem 6).

Proposition 1. If $u$ is one of the weak eigenfunctions corresponding to a weak eigenvalue $\lambda$, i.e., a non-trivial weak solution 
of (3)-(4), where

$$
p_{i j}=p_{j i}, q, \rho \in C_{0,0}(\bar{\Omega}) ; \partial \Omega \in \bar{C}_{1,0}
$$

and

$$
\sum_{i, j=1}^{m} p_{i j} \xi_{i} \xi_{j} \geqq 0\left(\left(\xi_{1}, \cdots, \xi_{m}\right) \text { is any real vector }\right) \text {, }
$$

then we have

$$
G_{o}[u]-\lambda H_{\Omega, \rho}[u]=0 .
$$

Proof. By the definition of a weak solution, $D_{o}[u]<\infty$, and there exists a sequence $\left\{u_{h} \in \stackrel{\circ}{W}_{2}^{(1)}(\Omega)\right\}$ such that

$$
D_{o}\left[u-u_{h}\right] \rightarrow 0, \quad\left\|u-u_{k}\right\|_{L_{2}(\rho)} \rightarrow 0,
$$

and for any $\zeta \in \stackrel{\circ}{W}_{2}^{(1)}(\Omega)$ we have

$$
G_{o}[u, \zeta]-\lambda H_{\rho, \rho}[u, \zeta]=0 .
$$

Putting $\zeta=u_{i}$, we get

$$
G_{\rho}\left[u, u_{h}\right]-\lambda H_{\rho_{, \rho}}\left[u, u_{h}\right]=0 .
$$

Therefore from Lemma 1 follows

$$
G_{o}[u]-\lambda H_{o, \rho}[u]=0 .
$$

Proposition 2. Assume that $p_{i j}=p_{j i}, q, \rho \in C_{0,0}(\bar{\Omega})$ and that $p_{i j}$ satisfy

$$
\sum_{i, j=1}^{m} p_{i j} \xi_{i} \xi_{j} \geqq 0\left(\left(\xi_{1}, \cdots, \xi_{m}\right) \text { is any real vector }\right) \text {. }
$$

Then for two weak eigenfunctions $u_{n}, u_{n^{\prime}}$ corresponding to weak eigenvalues $\lambda_{n} \neq \lambda_{n^{\prime}}$, we have

$$
H_{Q, \rho}\left[u_{n}, u_{n^{\prime}}\right]=0 \text {. }
$$

Proof. By the definition of a weak solution, $D_{o}\left[u_{n}\right], D_{o}\left[u_{n^{\prime}}\right]<\infty$, and there exist two sequences $\left\{u_{n h} \in \stackrel{\circ}{W}_{2}^{(1)}(\Omega)\right\},\left\{u_{n^{\prime} h} \in \stackrel{\circ}{W}_{2}^{(1)}(\Omega)\right\}$ such that

$$
\begin{aligned}
& D_{o}\left[u_{n}-u_{n h}\right] \rightarrow 0,\left\|u_{n}-u_{n h}\right\|_{L_{2}(Q)} \rightarrow 0 \\
& D_{\Omega}\left[u_{n^{\prime}}-u_{n^{\prime} h}\right] \rightarrow 0,\left\|u_{n^{\prime}}-u_{n^{\prime} h}\right\|_{L_{2}(Q)} \rightarrow 0
\end{aligned}
$$


and for any $\zeta \in \stackrel{\circ}{W}_{2}^{(1)}(\Omega)$ we have

$$
\begin{aligned}
& G_{Q}\left[u_{n}, \zeta\right]-\lambda_{n} H_{Q, \rho}\left[u_{n}, \zeta\right]=0, \\
& G_{o}\left[u_{n}, \zeta\right]-\lambda_{n}, H_{a, \rho}\left[u_{n^{\prime}}, \zeta\right]=0 .
\end{aligned}
$$

Without loss of generality we can assume $\lambda_{n} \neq 0$. Therefore from Lemma 1 follows

$$
\begin{aligned}
& H_{Q, \rho}\left[u_{n}, u_{n^{\prime}}\right]=\lim _{h \rightarrow \infty} H_{Q, \rho}\left[u_{n}, u_{n^{\prime} h}\right] \\
& =\lambda_{n}^{-1} \lim _{h \rightarrow \infty} G_{Q}\left[u_{n}, u_{n^{\prime} h}\right]=\lambda_{n}^{-1} G_{\rho}\left[u_{n}, u_{n^{\prime}}\right] \\
& =\lambda_{n}^{-1} \lim _{h \rightarrow \infty} G_{Q}\left[u_{n h}, u_{n^{\prime}}\right] \\
& =\lambda_{n}^{-1} \lambda_{n^{\prime}} \lim _{h \rightarrow \infty} H_{Q, \rho}\left[u_{n h}, u_{n^{\prime}}\right] . \\
& =\lambda_{n}^{-1} \lambda_{n^{\prime}} H_{\Omega, \rho}\left[u_{n}, u_{n^{\prime}}\right] .
\end{aligned}
$$

Since $\lambda_{n}^{-1} \lambda_{n^{\prime}} \neq 1$, we have

$$
H_{o, p}\left[u_{n}, u_{n^{\prime}}\right]=0
$$

Theorem 5. Assume that $p_{i j}=p_{j i}, q, \rho \in C_{0,0}(\bar{\Omega}) ; \partial \Omega \in \bar{C}_{1,0}$ and also that $p_{i j}$ and $\rho$ satisfy

$$
\begin{aligned}
& \sum_{i, j=1}^{m} p_{i j} \xi_{i} \xi_{j} \geqq p_{0}(x) \sum_{i=1}^{m} \xi_{i}^{2} \geqq 0 \\
& \quad\left(\left(\xi_{1}, \cdots, \xi_{m}\right) \text { is any real vector. }\right), \\
& \frac{1}{p_{0}} \in L_{k /(1-k)}(\Omega)
\end{aligned}
$$

(for some $k$ with $\frac{m}{m+2}<k<1$ when $m \geqq 2$, or with $\frac{1}{2}<k<1$ when $m=1$ )

$$
\rho \geqq \rho_{0}>0 \text {. }
$$

Then the weak spectrum of the eigenvalue problem for (3)-(4) are discrete, i.e., the totality of weak eigenvalues taken with respective multiplicity does not have any finite limiting point.

Proof. Suppose on the contrary that we had a sequence of eigenvalues $\left\{\lambda_{n}\right\}$ such that $\lim _{n \rightarrow \infty} \lambda_{n}=\lambda_{0} \neq \infty$. By Proposition 2 we can 
construct a system of weak eigenfunctions $\left\{u_{n}\right\}$ corresponding to $\left\{\lambda_{n}\right\}$, normalized and orthogonal with respect to $H_{a, p}[$,$] . From Proposition$ 1 follows $G_{\diamond}\left[u_{n}\right]=\lambda_{n}$.

Let $\varepsilon$ be any number such that $0<\varepsilon<\frac{1}{2}$. Then there exists an $n_{0}(\varepsilon)$ such that

$$
\lambda_{0}-\varepsilon<G_{0}\left[u_{n}\right]<\lambda_{0}+\varepsilon
$$

for every $n>n_{0}(\varepsilon)$. Since $u_{n}$ is a weak solution of $(3)-(4)$ for $\lambda=\lambda_{n}$, there exists $v_{n} \in \stackrel{\circ}{W}_{2}^{(1)}(\Omega)$ such that

$$
D_{\circ}\left[u_{n}-v_{n}\right]<\varepsilon, H_{o, \rho}\left[u_{n}-v_{n}\right]<\varepsilon,
$$

and therefore we have

$$
\begin{aligned}
& G_{o}\left[v_{n}\right]<\lambda_{0}+\eta(\varepsilon), \\
& H_{\rho_{p}}\left[v _ { n } \left[<(1+\sqrt{\varepsilon})^{2}\left(n>n_{0},(\varepsilon)\right),\right.\right.
\end{aligned}
$$

where $\eta(\varepsilon)$ is some positive number.

Because

$$
\begin{aligned}
& D_{\odot}\left[v_{n}\right] \leqq G_{\odot}\left[v_{n}\right]+\frac{\max _{\bar{\rho}}|q|}{\min _{\bar{\rho}} \rho} H_{\rho, \rho}\left[v_{n}\right] \\
& \leqq \lambda_{0}+\eta(\varepsilon)+\frac{\max _{\bar{D}}|q|}{\min _{\bar{\rho}} \rho}(1+\sqrt{\varepsilon})^{2}\left(n>n_{0}(\varepsilon)\right),
\end{aligned}
$$

from the corollary to Lemma 11 follows the relative compactness of $\left\{v_{n}\right\}$. Now take a Cauchy sequence in $L_{2}(\Omega)$ from $\left\{v_{n}\right\}$ and denote it again by $\left\{v_{n}\right\}$. Obviously

$$
H_{a, p}\left[v_{n}-v_{n^{\prime}}\right] \rightarrow 0 \quad\left(n, n^{\prime} \rightarrow \infty\right) .
$$

Whereas by the orthogonality of $\left\{u_{n}\right\}$ we have

$$
\begin{aligned}
& \sqrt{H_{Q, \rho}\left[v_{n}-v_{n^{\prime}}\right]}
\end{aligned}
$$

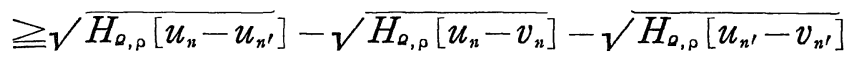

$$
\begin{aligned}
& \geqq \sqrt{H_{Q_{, \rho}}\left[u_{n}\right]-2 H_{Q_{\rho} \rho}\left[u_{n}, u_{n^{\prime}}\right]+H_{Q, \rho}\left[u_{n^{\prime}}\right]}-2 \sqrt{\bar{\varepsilon}} \\
& =\sqrt{2}(1-\sqrt{2 \varepsilon})\left(n>n_{0}(\varepsilon)\right) \text {. }
\end{aligned}
$$

Therefore we have 


$$
H_{a, p}\left[v_{n}-v_{n^{\prime}}\right] \nrightarrow 0 \quad\left(n, n^{\prime} \rightarrow \infty\right) .
$$

This is a contradiction.

Theorem 6. Assume that the bounded domain $\Omega$ in m-dimensional Euclidean space and the coefficients in (3)-(4) satisfy the following conditions:

$$
\begin{aligned}
& p_{i j}=p_{j i}, q, \rho \in C_{0, \alpha}(\Omega) \cap C_{0,0}(\bar{\Omega}), \\
& \frac{\partial p_{i j}}{\partial x_{j}} \in C_{0, \alpha}(\Omega)(i, j=1, \cdots, m), \\
& \partial \Omega \in \bar{C}_{2, \alpha}, \\
& \sum_{i, j=1}^{m} p_{i j} \xi_{i} \xi_{j} \geqq p_{0}(x) \sum_{i=1}^{m} \xi_{i}^{2} \geqq 0 \\
& \quad\left(\left(\xi_{1}, \cdots, \xi_{m}\right) \text { is any real vector. }\right), \\
& \quad \frac{1}{p_{0}} \in L_{k /(1-k)}(\Omega) \\
& \left(\text { for some } k \text { with } \frac{m}{m+2}<k<1 \text { when } m \geqq 2, \frac{1}{2}<k<1\right. \\
& \quad \text { when } m=1), \\
& \quad p_{0}>0 \quad \text { in } \Omega, \\
& \rho \geqq \rho_{0}>0 \quad \text { in } \bar{\Omega},
\end{aligned}
$$

(ii) the domain and the coefficients have Properties $(A)$ and $(B)$. Then there exist eigenfunctions $u_{1}, u_{2}, \cdots, u_{n}, \cdots\left(\in C_{2, \alpha}(\Omega) \cap C_{0,0}(\bar{\Omega})\right)$ corresponding to the eigenvalues $\lambda_{1} \leqq \lambda_{2} \leqq \cdots \leqq \lambda_{n} \leqq \cdots$ such that

$$
G_{\diamond}\left[u_{n}\right]=\inf _{\mathrm{u} \in \mathfrak{s}_{r_{o}}} G_{o}[u]=\lambda_{n}
$$

where

$$
\begin{gathered}
\mathfrak{W}_{n}=\left\{u \in \stackrel{\circ}{W}_{2}^{(1)}(\Omega) \mid H_{o, p}[u]=1, H_{a, p}\left[u, u \iota_{k}\right]=0\right. \\
(k=1, \cdots, n-1)\}
\end{gathered}
$$

Proof. Since this theorem is proved in the same way as Theorem 4 , suffice it to say that we give a rough proof.

Let $\left\{\Omega_{k}\right\}$ be an approximating sequence for $\Omega\left(\partial \Omega \in \bar{C}_{2, \alpha}\right)$. Consider the problem for $\Omega_{k}$, denote by $\mathfrak{W}_{n}\left(\Omega_{k}\right)$ the set of functions for $\Omega_{k}$ corresponding to $\mathfrak{W}_{n}$ for $\Omega$ and set 


$$
\begin{aligned}
& \inf _{u \in \mathfrak{W}_{n}} G_{o}[u]=\lambda_{n}, \\
& \inf _{\mathrm{u} \in \mathfrak{W}_{r_{0}}\left(\Omega_{k}\right)} G_{\varrho_{k}}[u]=\lambda_{n k} .
\end{aligned}
$$

It follows that $\lim _{k \rightarrow \infty} \lambda_{n k}=\lambda_{n}$. Now for any $k$ there exists an eigenfunction $u_{n k} \in C_{2, \alpha}\left(\Omega_{k}\right) \cap \stackrel{\circ}{W}_{2}^{(1)}(\Omega)$ such that

$$
G_{\Omega_{k}}\left[u_{n k}\right]=\inf _{\mathrm{u} \in \mathfrak{W}_{n_{3}}\left(\Omega_{k}\right)} G_{\varrho_{k}}[u]=\lambda_{n k},
$$

and therefore $G_{\varrho_{k}}\left[u_{n k}\right]$ is bounded. From this fact results the boundedness $D_{o}\left[u_{n k}\right]$, vrai $i_{\rho} \max \left|u_{n k}\right|$, and thereafter $\left\|u_{n k^{\prime}}\right\|_{c_{2, \alpha}\left(o_{k}\right)}\left(k^{\prime}>k\right)$.

By a diagonal process, we get a subsequence of $\left\{u_{n k}\right\}$ (hereafter we denote this subsequence again by $\left\{u_{n k}\right\}$ ) which converges in $C_{2,0}(\Omega)$ to a solution $u_{n}$ of (3) corresponding to $\lambda_{n}$. Using a strong barrier function we conclude that $u_{n}$ also satisfies (4). Obviously $D_{0}\left[u_{n}\right]<\infty$.

Finally we confirm that $u_{n}$ satisfies

$$
G_{\diamond}\left[u_{n}\right]=\inf _{u \in \mathfrak{M}_{\mathfrak{n}}} G_{o}[u]=\lambda_{n} .
$$

Since $u_{n}$ is an eigenfunction for (3)-(4) corresponding to $\lambda_{n}$, it follows from Proposition 1 that

$$
G_{o}\left[u_{n}\right]-\lambda_{n} H_{\Omega_{,}}\left[u_{n}\right]=0 .
$$

By the corollary to Lemma 11 for some subsequence $\left\{u_{n k}{ }^{\prime}\right\}$ of $\left\{u_{n k}\right\}$ we have

$$
\lim _{k \prime \rightarrow \infty} H_{o, \rho}\left[u_{n k^{\prime}}\right]=H_{Q, \rho}\left[u_{n}\right]
$$

Therefore

$$
\begin{aligned}
& H_{Q, \rho}\left[u_{n}\right]=\lim _{k^{\prime} \rightarrow \infty} H_{Q, \rho}\left[u_{n k^{\prime}}\right] \\
& =\lim _{k^{\prime} \rightarrow \infty} H_{\varrho_{k^{\prime}, \rho}}\left[u_{n k^{\prime}}\right]=\lim _{k^{\prime} \rightarrow \infty} 1=1 .
\end{aligned}
$$

Thus

$$
G_{\rho}\left[u_{n}\right]=\lambda_{n}
$$

\section{§. Lemmas}

In this section we shall state several lemmas, some of which were used in the proofs of the theorems in the preceding sections, while others are of more preliminary nature. Throughout this section 
we assume that $p_{i j}=p_{j i}, q, \rho, f \in C_{0,0}(\Omega)$ and $\partial \Omega \in \bar{C}_{1,0}$.

Lemma 1. Let $u, \quad v \in L_{2}(\Omega),\left\{u_{h} \in W_{2}^{(1)}(\Omega)\right\}$ and $\left\{v_{k} \in W_{2}^{(1)}(\Omega)\right\}$ be such that

$$
\begin{aligned}
& D_{o}[u]<\infty, D_{o}\left[u-u_{k}\right] \rightarrow 0,\left\|u-u_{h}\right\|_{L_{2}(o)} \rightarrow 0, \\
& D_{o}[v]<\infty, D_{o}\left[v-v_{r}\right] \rightarrow 0,\left\|v-v_{k}\right\|_{L_{2}(o)} \rightarrow 0,
\end{aligned}
$$

where $p_{i j}$ satisfies

$$
\sum_{i, j=1}^{m} p_{i j} \xi_{i} \xi_{j} \geqq 0\left(\left(\xi_{1}, \cdots, \xi_{m}\right) \text { is any real vector }\right) \text {. }
$$

Then we have

$$
\begin{aligned}
& D_{o}[u, v]=\lim _{h \rightarrow \infty} D_{S}\left[u_{h}, v\right]=\lim _{h, k \rightarrow \infty} D_{o}\left[u_{h}, v_{k}\right], \\
& (u, v)_{\rho}=\lim _{h \rightarrow \infty}\left(u_{h}, v\right)_{o}=\lim _{h, k \rightarrow \infty}\left(u_{h}, v_{k}\right)_{\odot},
\end{aligned}
$$

where $(u, v)_{0}=\int_{0} u v d V$.

Proof. From the assumption of the lemma follows

$$
D_{o}\left[u_{k}\right], D_{o}\left[v_{k}\right]<C,
$$

where $C$ does not depend on $h, k$. Obviously $D_{o}[u, v]<\infty$ and

$$
\begin{gathered}
D_{o}[u, v]=D_{o}\left[u-u_{h}, v-v_{k}\right]+D_{o}\left[u-u_{k}, v_{k}\right] \\
+D_{o}\left[u_{h}, v-v_{k}\right]+D_{o}\left[u_{k}, v_{k}\right] .
\end{gathered}
$$

Letting $h, k \rightarrow \infty$, we get

$$
D_{o}[u, v]=\lim _{h, k \rightarrow \infty} D_{o}\left[u_{h}, v_{k}\right] .
$$

In a similar way we have

$$
D_{o}[u, v]=\lim _{h \rightarrow \infty} D_{o}\left[u_{h}, v\right] \text {. }
$$

The equalities for $(,)_{0}$ are obvious.

Lemma 2. If $u \in \stackrel{\varphi}{W}_{2}^{(1)}(\Omega)$ and $K \geqq \max _{\partial \rho} \varphi$, then $u^{(K)} \in \stackrel{\circ}{W}_{2}^{(1)}(\Omega)$

(Cf. Ladyzhenskaya-Ural'tseva [3] p. 75, Lemma 3.3).

Lemma 3. If $u \in \stackrel{\circ}{W}_{2}^{(1)}(\Omega)$, then

$$
\|u\|_{\Sigma_{2 k n /(m-2 k)(\Omega)} \leqq} \frac{k(m-1)}{m-2 k}\|\nabla u\|_{L 2 k(\Omega)}\left(\frac{1}{2}<k<\frac{m}{2}\right) .
$$


Proof. When $u \in \stackrel{\circ}{W}_{2}^{(1)}(\Omega)$, we have

$$
\|u\|_{L m /(m-1)(\rho)} \leqq \frac{1}{2}\|\nabla u\|_{L_{1}(\rho)}(m \geqq 2)
$$

(cf. Nirenberg [5] p. 14). If we put $u=u^{\left.2 k(m-1)^{\prime m-2 k}\right)}$, then we get the inequality of the lemma (more precisely we must show in the first place that we have this inequality for $u \in \dot{C}_{\infty}(\Omega)$, and in the next place that we have it for $\left.u \in \stackrel{\circ}{W}_{2}^{(1)}(\Omega)\right)$.

Lemma 4. For any $u \in \stackrel{\circ}{W_{2}^{(1)}}(\Omega)$ we have

$$
\|u\|_{L_{2}(\varrho)} \leqq B_{m k}\|\nabla u\|_{L_{2 k}(\varrho)},
$$

where

$$
B_{m k}= \begin{cases}\frac{k(m-1)}{m-2 k}(\operatorname{mes} \Omega)^{\{(m+2) k-m\} / 2 k m} & (m \geqq 2) \\ \frac{1}{2}(\operatorname{mes} \Omega)^{(3 k-1) / 2 k} & (m=1)\end{cases}
$$

and

$$
\begin{array}{cc}
\frac{m}{m+2}<k<\frac{m}{2} & (m \geqq 2) \\
k>\frac{1}{2} & (m=1) .
\end{array}
$$

Proof. When $m \geqq 2$, by Hölder's inequality we have

$$
\|u\|_{L_{2}(Q)} \leqq\|u\|_{L_{2 k m /(m-2 k,(Q)}(\operatorname{mes} \Omega)^{\{(m+2) k-m\}^{\prime} 2 m k}} .
$$

Therefore from Lemma 3 follows the inequality of the lemma.

When $m=1$, by the inequality

$$
|u(x)| \leqq \frac{1}{2} \int_{-\infty}^{\infty}|\nabla u| d V
$$

and Hölder's inequality we have

$$
|u| \leqq \frac{1}{2}(\operatorname{mes} \Omega)^{(2 k-1) / 2 k}\left\|_{i} \nabla u\right\|_{L_{2 k}(Q)} .
$$

Therefore we get the inequality of the lemma.

Corollary 1. If $u \in \stackrel{\Phi}{W}{ }_{2}^{(1)}(\Omega)$, then for any $K \geqq \max _{\partial \rho} \varphi$ we have 


$$
\left\|u^{(K)}\right\|_{L_{2}\left(\Omega^{(K)}\right)} \leqq B_{m k}^{\prime}\|\nabla\|_{L_{2 k}\left(\Omega^{(K)}\right)},
$$

where

$$
B_{m, k}^{\prime}= \begin{cases}\frac{k(m-1)}{m-2 k}\left(\operatorname{mes} \Omega^{(K)}\right)^{[(m+2) k-m\} / 2 k m} & (m \geqq 2) \\ \frac{1}{2}\left(\operatorname{mes} \Omega^{(K)}\right)^{(3 k-1) / 2 k} & (\mathrm{~m}=1)\end{cases}
$$

and

$$
\begin{array}{ll}
\frac{m}{m+2}<k<\frac{m}{2} & (m \geqq 2) \\
k>\frac{1}{2} & (m=1) .
\end{array}
$$

This inequality follows from Lemma 2 and Lemma 4.

Corollary 2. For any $u \in \stackrel{\circ}{W}_{2}^{(1)}(\Omega)$ we have

$$
\|u\|_{L_{2 k}(\rho)} \leqq B_{m k}^{\prime \prime}\|\nabla u\|_{L_{2 k}(Q)}
$$

where

$$
B_{m k}^{\prime \prime}= \begin{cases}\frac{k(m-1)}{m-2 k}(\operatorname{mes} \Omega)^{1 / m} & (m \geqq 2), \\ \frac{1}{2} \operatorname{mes} \Omega & (m=1)\end{cases}
$$

and

$$
\begin{array}{cc}
\frac{1}{m+2}<k<\frac{m}{2} & (m \geqq 2), \\
k>\frac{1}{2} & (m=1) .
\end{array}
$$

This inequality follows from Hölder's inequality and Lemma 4.

Lemma 5. For any $u \in \stackrel{\circ}{W}_{2}^{(1)}(\Omega)$ we have

$$
\begin{gathered}
\|u\|_{L_{2}(\rho)}^{2} \leqq\left(\frac{k(m-1)}{m-2 k}\right)^{m(1-k) / k}\|u\|_{L_{2 k}(\rho)}^{(m+2) k-m] / k} \|\left[\nabla u \|_{L_{2 k}(\rho)}^{m(1-k) / k}\right. \\
\left(m \geqq 2, \frac{m}{m+2}<k<1\right)
\end{gathered}
$$

or we have 


$$
\begin{gathered}
\|u\|_{L_{2}(\rho)}^{2} \leqq\left.\left\{\frac{1}{2}(\text { mes } \Omega)^{(2 k-1) / 2 k}\right\}^{2(1-k)}\|u\|\right|_{L_{2 k}(\rho)} ^{2 k}\|\nabla u\|_{L_{2 k}(\rho)}^{2(1-k)} \\
\left(m=1, \frac{1}{2}<k<1\right) .
\end{gathered}
$$

Proof. We begin with the case $m \geqq 2$. From Hölder's inequality follows

$$
\begin{aligned}
& \int_{0} u^{2} d V=\int_{0} u^{\alpha} u^{2-\alpha} d V \\
& \leqq\left[\int_{0} u^{2 k} d V\right]^{\alpha / 2 k}\left[\int_{\sigma} u^{(2-\alpha) \cdot 2 k / 2 k-\alpha} d V\right]^{(2 k-\alpha) / 2 k}
\end{aligned}
$$

for every $\alpha(0<\alpha<2 k)$. Putting $\alpha=\{(m+2) k-m\} / k$, we have

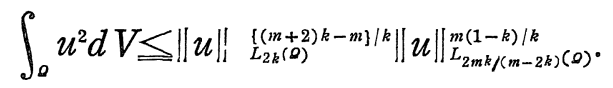

Therefore from Lemma 3 follows the inequality of the lemma.

When $m=1$, by the inequality (5) in the proof of Lemma 4 we get

$$
\begin{aligned}
& \int_{0} u^{2} d V=\int_{0} u^{2 k} u^{2-2 k} d V \\
& \quad \leqq \int_{0} u^{2 k} d V\left\{\frac{1}{2}(\operatorname{mes} \Omega)^{(2 k-1) / 2 k}\left\|_{\|} \nabla u\right\|_{L_{2 k}(\Omega)}\right\}^{2(1-k)} \\
& \leqq\left\{\frac{1}{2}(\operatorname{mes} \Omega)^{(2 k-1) / 2 k}\right\}^{2(1-k)}\|u\|_{L_{2 k}(Q)}^{2 k}\|\nabla u\|_{L_{2 k}(\Omega)}^{2(1-k)}
\end{aligned}
$$

Lemma 6. If $u \in \stackrel{\Phi}{W_{2}^{(1)}}(\Omega)$ satisfies

$$
\int_{Q^{(K)}}(u-K) d V \leqq C K\left(\operatorname{mes} \Omega^{(K)}\right)^{1+\varepsilon}
$$

for some positive $\varepsilon$ and for every $K \geqq K_{0}>0$, then

$$
\underset{o}{\operatorname{vrai}} \max u \leqq C^{\prime}
$$

where

$$
C^{\prime}=\left[K_{0}^{1 /(1+\varepsilon)}+C^{1 /(1+\varepsilon)}\left\{K_{0} \operatorname{mes} \Omega^{\left(K_{0}\right)}+\|u\|_{L_{1}\left(\Omega^{\left(K_{0}\right)}\right)}\right\}^{\varepsilon /(1+\varepsilon)}\right]^{(1+\varepsilon) / \varepsilon}
$$

(Cf. Ladyzhenskaya-Ural'tseva [3] p. 92, Lemma 5.1)

Lemma 7. If $u \in \stackrel{\varphi}{W}_{2}^{(1)}(\Omega)$ satisfies 


$$
\begin{aligned}
\|\nabla u\|_{L_{2 k}\left(Q^{(K)}\right)} \leqq C K\left(\operatorname{mes} \Omega^{(K)}\right)^{1 / 2} & \\
& \left(\frac{m}{m+2}<k<\frac{m}{2}, m \geqq 2\right)
\end{aligned}
$$

for every $K \geqq K_{0}>0$, then

$$
\text { vari } \max u<C^{\prime},
$$

where $C^{\prime}$ corresponds to $C^{\prime}$ in Lemma 6 , in which we replace $\varepsilon$ by $\frac{(m+2) k-m}{2 m k}, C$ by $\frac{k(m-1)}{m-2 k} C$, and $K_{0}$ by $\max \left\{K_{0}, \max _{\partial \rho} \varphi\right\}$.

Proof. By Schwartz's inequality we have

$$
\int_{Q(K)}(u-K) d V \leqq\left\|u^{(K)}\right\|_{L_{2}\left(\Omega^{\left.(K)_{\rho}\right)}\right.}\left(\operatorname{mes} \Omega^{(K)}\right)^{1 / 2} .
$$

Therefore from Corollary 1 to Lemma 4 and the assumption of the present lemma follows

$$
\int_{Q^{(K)}}(u-K) d V \leqq \frac{k(m-1)}{m-2 k} C K\left(\operatorname{mes} \Omega^{(K)}\right)^{1+\{(m+2) k-m\} / 2 m k}
$$

for every $K \geqq \max \left\{K_{0}, \max _{\partial o} \varphi\right\}$. Hence the assumption of Lemma 6 is satisfied.

Lemma 8. Assume

$$
\begin{aligned}
\sum_{i, j=1}^{m} p_{i j} \xi_{i} \xi_{j} \geqq p_{0}(x) \sum_{i=1}^{m} \xi_{i}^{2} \geqq 0 \\
\left(\left(\xi_{1}, \cdots, \xi_{m}\right) \text { is any real vector }\right)
\end{aligned}
$$

and

$$
\frac{1}{p_{0}} \in L_{k /(1-k)}(\Omega)(0<k<1) .
$$

Then for any $u \in \stackrel{\circ}{W}_{2}^{(1)}(\Omega)$, we have

$$
\|\nabla u\|_{L_{2 k}(\rho)}^{2} \leqq\left\|\frac{1}{p_{0}}\right\|_{L_{k / 1(-k)(\Omega)}} D_{o}[u]
$$

Proof. From Hölder's inequality and from the assumption of the lemma follows

$$
\|\nabla u\|_{L_{2 k}(\rho)}^{2}=\left[\int_{\varrho}\left(\frac{1}{p_{0}}\right)^{k}\left\{\sum_{i=1}^{m} p_{0}\left(\frac{\partial u}{\partial x_{i}}\right)^{2}\right\}^{k} d V\right]^{1 / k}
$$




$$
\leqq\left\|1 / p_{0}\right\|_{L_{k /(1-k)}(\rho)} \int_{0} \sum_{i, j=1}^{m} p_{i j}\left(\frac{\partial u}{\partial x_{i}}\right)\left(\frac{\partial u}{\partial x_{j}}\right) d V .
$$

Corollary 1. Under the same assumption as in the lemma, if $u \in \stackrel{\Phi}{W}_{2}^{(1)}(\Omega)$, we have

$$
\|\nabla u\|_{L_{2 k}(Q(K))}^{2} \leqq\left\|\frac{1}{p_{0}}\right\| \|_{\left.L_{k / 1}-k\right)(Q)} D_{Q(K)}[u]
$$

for every $K \geqq \max _{\partial \rho} \varphi$.

This inequality follows from Lemma 2 and Lemma 8.

Corollary 2. Under the same assumption as in the lemma, if $u \in \stackrel{\Phi}{W}_{2}^{(1)}(\Omega)$, we have

$$
\left\|u^{(K)}\right\|_{L_{2}\left(G_{(S)}(K)\right.} \leqq B_{m l \cdot}^{\prime} \sqrt{\left\|\frac{1}{p_{0}}\right\|_{L_{k / 1-k)(Q)}} D_{Q(K)}[u]}
$$

for every $K \geqq \max \varphi$, where $B_{m k}^{\prime}$ and $k$ are the same constants as in Corollary 1 to Lemma 4.

In fact, by Corollary 1 to Lemma 4 and Corollary 1 to Lemma 8 we get the desired inequality.

Lemma 9. Assume

$$
\begin{gathered}
\sum_{i, j=1}^{m} p_{i j} \xi_{i} \xi_{j} \geqq p_{0}(x) \sum_{i=1}^{m} \xi_{i}^{2} \geqq 0 \\
\left(\left(\xi_{1}, \cdots, \xi_{m}\right) \text { is any real vector }\right), \\
\frac{1}{p_{0}} \in L_{k /(1-k)}(\Omega) \quad\left(\text { for some } k \text { with } \frac{m}{m+1}<k<1 \text { when } m \geqq 2\right. \text { or } \\
\text { with } \left.\frac{1}{2}<k<1 \quad \text { when } m=1\right) \\
q \geqq 0,
\end{gathered}
$$

and

$$
f \in C_{0,0}(\bar{\Omega})
$$

Then we have

$$
\inf _{\substack{\Phi \\ u \in W_{2}^{(1)}(\Omega)}}\left(G_{\Omega}[u]+2 H_{\Omega, 1}[f, u]\right)>-\infty .
$$

Proof. Let $K$ be a constant larger than $\max _{\partial \rho} \varphi$. Then we have 


$$
\begin{gathered}
G_{o}[u]+2 H_{o, 1}[f, u] \\
\geqq \int_{\rho} \sum_{i, j=1}^{m} p_{i j} \frac{\partial u}{\partial x_{i}} \frac{\partial u}{\partial x_{j}} d V+\int_{o} q u^{2} d V-2 \int_{o}\left(u^{(K)}+K\right)|f| d V \\
\geqq \int_{\rho^{(K)}} \sum_{i, j=1}^{m} p_{i j} \frac{\partial u}{\partial x_{i}} \frac{\partial u}{\partial x_{j}} d V-\varepsilon \int_{o(K)}\left\{u^{(K)}\right\}^{2} d V \\
-\frac{1}{\varepsilon} \int_{\rho} f^{2} d V-2 K \max _{\rho}|f| \operatorname{mes} \Omega(\varepsilon>0) .
\end{gathered}
$$

By Corollary 1 to Lemma 4 and Corollary 1 to Lemma 8, we get

$$
\begin{aligned}
G_{Q}[u] & +2 H_{\rho, 1}[f, u] \\
& \geqq\left\{\frac{1}{\left\|\frac{1}{p_{0}}\right\|_{\left.L_{k / 1}-k\right)}(\rho)}-{ }_{\varepsilon} B_{m k}^{\prime 2}\right\}\|\nabla u\|_{L_{2 k}\left(\Omega^{(K)}\right)}^{2} \\
& -\left(\frac{1}{\varepsilon} \max _{\rho}|f|+2 K\right) \max _{\Omega}|f| \operatorname{mes} \Omega .
\end{aligned}
$$

Therefore, by choosing $\varepsilon$ so small that the coefficient of $\|\nabla u\|_{\left.L_{2 k} \mathcal{Q}^{(K)}\right)}^{2}$ is positive, we have

$$
\begin{aligned}
G_{\Omega}[u] & +2 H_{\rho, 1}[f, u] \\
& \geqq-\left(\frac{1}{\varepsilon} \max _{\bar{\rho}}|f|+2 K\right) \max _{\Omega}|f| \operatorname{mes} \Omega .
\end{aligned}
$$

Lemma 10. Under the same assumption as in Lemma 9, if $u \in$ $\stackrel{\Phi}{W}_{2}^{(1)}(\Omega)$ satisfies

$$
G_{o}[u]+2 H_{o, 1}[f, u] \leqq C,
$$

then we have

$$
D_{\diamond}[u] \leqq G_{o}[u] \leqq C^{\prime} \text {, }
$$

where $C^{\prime}$ depends on $m, k, C,\left\|1 / p_{0}\right\|_{L_{k /(1-k)(\rho)},} \max _{\Omega^{-}}|f|, \max _{\partial \Omega} \varphi$ and $\operatorname{mes} \Omega$.

Proof. Let $K$ be a constant larger than $\max _{\partial \rho} \varphi$. Then we have

$$
\begin{aligned}
& G_{\rho}[u] \leqq C+2 \int_{\Omega}|f|\left(u^{(K)}+K\right) d V \\
& \quad \leqq C+2 \int_{Q^{(K)}}|f| u^{(K)} d V+2 K \max _{\bar{\rho}}|f| \operatorname{mes} \Omega \\
& \quad \leqq C+\left(\frac{1}{\varepsilon} \max _{\Omega}|f|+2 K\right) \max _{\bar{\rho}}|f| \operatorname{mes} \Omega+\varepsilon \int_{\Omega^{(K)}}\left\{u^{(K)}\right\}^{2} d V .
\end{aligned}
$$

From Corollary 2 to Lemma 8 follows 


$$
\begin{aligned}
& G_{\varrho}[u] \leqq C+\left(\frac{1}{\varepsilon} \max _{\bar{\rho}}|f|+2 K\right) \max _{\bar{\rho}}|f| \operatorname{mes} \Omega \\
& +\varepsilon B_{m k}^{\prime 2}\left\|\frac{1}{p_{0}}\right\|_{L_{k} /(1-k)}^{(\ell)} D_{\varrho^{(K)}}[u] \\
& \leqq C+\left(\frac{1}{\varepsilon} \max _{\overline{\bar{D}}}|f|+2 K\right) \max _{\overline{\bar{\rho}}}|f| \operatorname{mes} \Omega \\
& +\varepsilon B_{m k}^{\prime 2}\left\|\frac{1}{p_{0}}\right\|_{L_{k /(1-k)(\rho)}} G_{\ell}[u] .
\end{aligned}
$$

Therefore, by choosing $\varepsilon$ so small that the coefficient of $G_{o}[u]$ is less than $\frac{1}{2}$, we have

$$
G_{\iota}[u] \leqq 2 C+2\left(\frac{1}{\varepsilon} \max _{\overline{\bar{D}}}|f|+2 K\right) \max _{\overline{\bar{D}}}|f| \operatorname{mes} \Omega \text {. }
$$

Lemma 9'. Assume

$$
\sum_{i, j=1}^{m} p_{i j} \xi_{i} \xi_{j} \geqq 0\left(\left(\xi_{1}, \cdots, \xi_{m}\right) \text { is any real vector }\right)
$$

and

$$
\rho \geqq \rho_{0}>0 \text {. }
$$

Then

$$
\inf _{u \in \mathfrak{W}_{1}} G_{2}[u]>-\infty,
$$

where $\mathfrak{W}_{1}=\left\{u \in \stackrel{\circ}{W}_{2}^{(1)}(\Omega) \mid H_{\Delta, p}[u]=1\right\}$.

Proof.

$$
\begin{aligned}
G_{\Omega}[u] & \geqq \int_{\rho} \sum_{i, j=1}^{m} p_{i j} \frac{\partial u}{\partial x_{i}} \frac{\partial u}{\partial x_{j}} d V+\int_{\varrho} q u^{2} d V \\
& \geqq-\max _{\bar{\rho}} \frac{|q|}{\rho} H_{Q, \rho}[u]=-\max _{\overline{\bar{D}}} \frac{|q|}{\rho} .
\end{aligned}
$$

Lemma 10'. Under the same assumption as in Lemma 9', if $u \in \mathfrak{W}_{1}$, then we have

$$
D_{o}[u] \leqq G_{o}[u]+\max _{\bar{\rho}} \frac{|q|}{\rho} .
$$

Proof.

$$
D_{o}[u] \leqq G_{o}[u]+\left|H_{o, q}[u]\right|
$$




$$
\leqq G_{\rho}[u]+\max _{\bar{\rho}} \frac{|q|}{\rho} H_{o, \rho}[u]
$$

Lemma 11. Any bounded set in $\stackrel{\circ}{W}_{p}^{(1)}(\Omega)$ is relatively compact in $L_{p}(\Omega)$.

(Cf. Smirnov [8] p. 351.3)

Corollary. Assume

$$
\begin{aligned}
& \sum_{i, j=1}^{m} p_{i} \xi_{i} \xi_{j} \geqq p_{0}(x) \sum_{i=1}^{m} \xi_{i}^{2} \geqq 0 \\
& \left(\left(\xi_{1}, \cdots, \xi_{m}\right) \text { is any real vector }\right) \text {, } \\
& \frac{1}{p_{0}} \in L_{k / 1-k)}(\Omega) \quad \text { (for some } k \text { with } \frac{m}{m+2}<k<1 \\
& \text { when } m \geqq 2 \text {, or with } \frac{1}{2}<k<1 \text { when } m=1 \text { ). }
\end{aligned}
$$

Then

$$
\mathfrak{A}=\left\{u \in \stackrel{\circ}{W}_{2}^{(1)}(\Omega) \mid D_{s}[u]<C\right\}
$$

is relatively compact in $L_{2}(\Omega)$.

Proof. From the assumption of the corollary and from Lemma 8 follows $\|\nabla u\|_{L_{2 k}(\rho)}<C^{\prime}$, and therefore from Corollary 2 to Lemma 4 follows $\|u\|_{L_{2 k}(\rho)}<C^{\prime \prime}$. Thus by Lemma $11 \mathfrak{A}$ is relatively compact in $L_{2 k}(\Omega)$.

New Let $\left\{u_{n}\right\}$ be a Cauchy sequence in $L_{2 k}(\Omega)$. Since

$$
\begin{aligned}
& \left\|\nabla\left(u_{n}-u_{n^{\prime}}\right)\right\|_{L_{2 k}(e)} \\
& \quad \leqq\left\|\nabla u_{n}\right\|_{L_{2 k}(e)}+\left\|\nabla u_{n^{\prime}}\right\|_{L_{2 k}(e)}<2 C^{\prime}
\end{aligned}
$$

and

$$
\left\|u_{n}-u_{n^{\prime}}\right\|_{L_{2 k}(\rho)} \rightarrow 0 \quad\left(n, n^{\prime} \rightarrow \infty\right),
$$

from Lemma 5 follows

$$
\left\|u_{n}-u_{n^{\prime}}\right\|_{L_{2}(\Omega)} \rightarrow 0 \quad\left(n, n^{\prime} \rightarrow \infty\right),
$$

so that $\left\{u_{n}\right\}$ is also a Cauchy sequence in $L_{2}(\Omega)$. Hence $\mathfrak{A}$ is rela-

3) In [8] only the relative compactness in $L_{p}\left(\Omega^{\prime}\right)\left(\Omega^{\prime}\right.$ is strictly contained in $\Omega$ ) are stated. When $u \in \stackrel{\circ}{W}_{p}^{(1)}(\Omega)$, a similar discussion leads to the relative compactness in $L_{p}(\Omega)$ (cf. Suzuki [9], p. 44). 
tively compact in $L_{2}(\Omega)$.

Lemma 12. Let the elliptic operator

$$
L u \equiv \sum_{i, j=1}^{m} \frac{\partial}{\partial x_{j}}\left(p_{i j} \frac{\partial u}{\partial x_{i}}\right)-q u
$$

be such that

$$
\begin{gathered}
\sum_{i, j=1}^{m} p_{i j} \xi_{i} \xi_{j} \geqq p_{0} \sum_{i=1}^{m} \xi_{i}^{2} \\
\left(p_{0}>0,\left(\xi_{1}, \cdots, \xi_{m}\right) \text { is any real vector }\right) \\
\frac{\partial p_{i j}}{\partial x_{i}}, q \in C_{0, \alpha}(\bar{\Omega}) . \\
\text { If } u \in C_{2, \alpha(\bar{\Omega}), \text { then we have }} \\
\|u\|_{c_{2, \alpha}\left(\bar{s}^{\prime}\right)} \leqq C\left[\|L u\|_{c_{0, \alpha}(\bar{\Omega})}+\max _{\overline{\bar{\Omega}}} \mid u \|\right]
\end{gathered}
$$

where $\Omega^{\prime}$ is any subdomain of $\Omega$ which is strictly contained in $\Omega$, and $C$ depends on $\alpha,\left\|\frac{\partial p_{i j}}{\partial x_{i}}\right\|\left\|_{c_{0 \alpha},(\bar{Q})},\right\| q \|_{c_{o, \alpha}(\bar{\rho}),} p_{0}$, the diameter of $\Omega$, and the distance between $\Omega^{\prime}$ and $\partial \Omega$ (Cf. Schauder [7]).

\section{§. Property (A) and Property (B)}

In the first place we shall discuss a sufficient condition for the domain and the coefficients in the problem (1)-(2) to have Property (A).

Assume

$$
p_{i j} \in C_{1,0}(\Omega) \cap C_{0,0}(\bar{\Omega}) ; q, f \in C_{0,0}(\bar{\Omega}) ; \varphi \in C_{0,0}(\partial \Omega)
$$

and

$$
\sum_{i, j=1}^{m} p_{i j} \xi_{i} \xi_{j} \geqq 0\left(\left(\xi_{1}, \cdots, \xi_{m}\right) \text { is any real vector }\right) \text {. }
$$

If $q>0$ in $\Omega$ and $v \in C_{2,0}(\Omega) \cap C_{0,0}(\bar{\Omega})$ satisfies

$$
\begin{aligned}
& L[v] \leqq \min _{o} f \quad \text { in } \Omega \\
& v \geqq \max _{\partial \Omega} \varphi \text { on } \partial \Omega
\end{aligned}
$$

resp. 


$$
\begin{array}{ll}
L[v] \geqq \max _{\bar{a}} f & \text { in } \Omega \\
v \leqq \min _{\partial \Omega} \varphi & \text { on } \partial \Omega,
\end{array}
$$

then by the well known maximum principle, we have

$$
u \leqq v \quad \text { in } \bar{\Omega}
$$

resp.

$$
u \geqq v \quad \text { in } \bar{\Omega}
$$

for any solution $u$ of the problem (1)-(2) (cf. Courant-Hilbert [1], p. 329).

Suppose on the other hand $q \ngtr 0$ at $x^{\prime} \in \bar{\Omega}$. If there exists $i_{0}$ such that $p_{i_{0 i} 0}\left(x^{\prime}\right)>0$ and $\frac{\partial p_{i_{0} j}}{\partial x} \in C_{0,0}(\bar{\Omega})(j=1, \cdots, m)$, then there exists a subdomain $\Omega^{\prime}$ of $\Omega$ such that $\overline{\Omega^{\prime}} \ni x^{\prime}$ and

$$
\begin{aligned}
& a-e^{-b\left(x_{i_{0}}-x^{\prime} i_{0}\right)}>0, \\
& b e^{-b\left(x_{i_{0}}-x^{\prime} i_{0}\right)}\left(\left(p_{i_{0} \cdot 0} b-\sum_{j=1}^{m} \frac{\partial p_{i 0 j}}{\partial x_{j}}\right)+q\left(a-e^{-b\left(x_{10}-x^{\prime} i_{0}\right)}\right)>0\right.
\end{aligned}
$$

in $\Omega^{\prime}$, for some positive constants $a, b$. Hence by the transformation

$$
u=\left(a-e^{-b\left(x_{i 0}-x^{\prime} i_{0}\right)}\right) w,
$$

the problem is reduced to the case where $q>0$ in $\Omega^{\prime}$.

Therefore, if $\partial \Omega=\Gamma_{0} \cup \Gamma_{1} \cup \cdots \cup \Gamma_{m}$, then domain $\Omega$ and the coeffcients in the problem have Property (A), where

$$
\begin{aligned}
& \Gamma_{0}=\{x \in \partial \Omega \mid q(x)>0\}, \\
& \Gamma_{i}=\left\{x \in \partial \Omega \mid p_{i i}(x)>0, \text { and } \frac{\partial p_{i j}}{\partial x_{j}}(j=1, \cdots, m)\right.
\end{aligned}
$$

are continuous in a neighbourhood of $x$.

We now turn to the discussion of Property (B). Let $x_{0}$ be a boundary point, and $x_{0} \in S$, where (i) $S$ belongs to $C_{2,0}(\partial \Omega$ need not belong to $\left.C_{2,0}\right)$, i. e., there exist a neighbourhood $U_{x_{0}}=\left\{x|| x-x_{0} \mid<\sigma\right\}$ and the local coordinates $y_{1}(x), \cdots, y_{m}(x) \in C_{2,0}\left(U_{x_{0}}\right)$ such that

$$
U_{x_{0}} \cap S=\left\{x \in U_{x_{0}} \mid y_{m}(x)=y_{m}\left(x_{0}\right)=0\right\}
$$

and 


$$
\left|\frac{D\left(y_{1}, \cdots, y_{m}\right)}{D\left(x_{1}, \cdots, x_{m}\right)}\right| \neq 0 \text { in } U_{x_{0}}
$$

and (ii) $S$ satisfies

$$
U_{x_{0}} \cap \Omega \subset\left\{x \in U_{x_{0}} \mid y_{m}(x)>y_{m}\left(x_{0}\right)=0\right\} .
$$

Assume, rewriting $U_{x_{0} \cap \Omega}$ as $\omega\left(x_{0}, \sigma\right)$, that

$$
\begin{aligned}
& \left.p_{i j} \in C_{1,0}\left(\omega\left(x_{0}, \sigma\right)\right) \cap C_{0,0}\left(\overline{\omega\left(x_{0}, \sigma\right.}\right)\right), \\
& q \in C_{0,0}\left(\overline{\omega\left(x_{0}, \sigma\right)}\right), \\
& \sum_{i, j=1}^{m} \frac{\partial}{\partial x_{j}}\left(p_{i j} \frac{\partial y_{k}}{\partial x_{j}}\right)\left(y_{k}-y_{k}\left(x_{0}\right)\right)((k=1, \cdots, m-1) \text { is bounded } \\
& \left.\quad \text { in } \omega\left(x_{0}, \sigma\right)\right)
\end{aligned}
$$

and that there exist constants $a, b, c(0<a<1, b>0, c>0)$ such that

$$
\begin{aligned}
& (a-1) \sum_{i, j=1}^{m} p_{i j} \frac{\partial y_{m}}{\partial x_{i}} \frac{\partial y_{m}}{\partial x_{j}} y_{m}^{a-2}+\sum_{i, j=1}^{m} \frac{\partial}{\partial x_{j}}\left(p_{i j} \frac{\partial y_{m}}{\partial x_{i}}\right) y_{m}^{a-1} \\
& <-b y_{m}^{-c} \text { in } \omega\left(x_{0}, \sigma\right) .
\end{aligned}
$$

Then, by setting

$$
v=\sum_{i=1}^{m-1}\left(y_{i}-y_{i}\left(x_{0}\right)\right)^{2}+y_{m}^{a},
$$

we can construct a strong barrier function.

In fact, taking $\sigma$ sufficiently small, we have

$$
\begin{aligned}
& \quad L[v]=\sum_{i, j=1}^{m} \frac{\partial}{\partial x_{j}}\left(p_{i j} \frac{v v}{x_{i}}\right)-q v \\
& =\sum_{i, j=1}^{m} \sum_{k, l=1}^{m} p_{i j} \frac{\partial y_{k}}{\partial x_{i}} \frac{\partial y_{l}}{\partial x_{j}} \frac{\partial^{2} v}{\partial y_{k} \partial y_{l}}+\sum_{i, j=1}^{m} \sum_{k=1}^{m} \frac{\partial}{\partial x_{j}}\left(p_{i j} \frac{\partial y_{k}}{\partial x_{i}}\right) \frac{\partial v}{\partial y_{k}}-q v \\
& =2 \sum_{k=1}^{m-1} \sum_{i, j=1}^{m} p_{i j} \frac{\partial y_{k}}{\partial x_{i}} \frac{\partial y_{k}}{\partial x_{j}}+2 \sum_{k=1}^{m-1} \sum_{i, j=1}^{m} \frac{\partial}{\partial x_{j}}\left(p_{i j} \frac{\partial y_{k}}{\partial x_{j}}\right)\left(y_{k}-y_{k}\left(x_{0}\right)\right)-q v \\
& +a(a-1) \sum_{i, j=1}^{m} p_{i j} \frac{\partial y_{m}}{\partial x_{i}} \frac{\partial y_{m}}{\partial x_{j}} y_{m}^{a-2}+a \sum_{i, j=1}^{m} \frac{\partial}{\partial x_{j}}\left(p_{i j} \frac{\partial y_{m}}{\partial x_{i}}\right) y_{m}^{a-1} \\
& <0 \text { in } \omega\left(x_{0}, \sigma\right) .
\end{aligned}
$$

Obviously $v$ satisfies all other conditions for a strong barrier function.

\section{$\S 7 . \quad$ Examples}

1) Let

$$
\Omega=\left\{x \mid \sum_{k=1}^{m} x_{k}^{2}<1(m \geqq 2)\right\}
$$


Consider the problem

$$
\begin{array}{cc}
\sum_{i=1}^{m} \frac{\partial}{\partial x_{i}}\left\{\left(1-\sqrt{\sum_{k=1}^{m} x_{k}^{2}}\right)^{2 / m-\varepsilon_{i}} \frac{\partial u}{\partial x_{i}}\right\}-q u & =f\left(0<\varepsilon_{i} \leqq 2 / m\right) \\
& \text { in } \Omega, \\
& \text { on } \partial \Omega,
\end{array}
$$

where

$$
\begin{aligned}
& q, f \in C_{0, \alpha}(\Omega) \cap C_{0,0}(\bar{\Omega}), \\
& \varphi \in C_{2, \alpha}(\Omega) \cap C_{0,0}(\bar{\Omega}),
\end{aligned}
$$

and

$$
q \geqq 0 \text { in } \bar{\Omega}(q \neq 0 \text { on } \partial \Omega) .
$$

This problem has a unique solution.

Proof. Here suffice it to say that we prove that Property (B) is satisfied. Let $x_{0} \in \partial \Omega$. In $\omega\left(x_{0}, \sigma\right)$ take $y_{l}=1-\sqrt{\sum_{k=1}^{l} x_{k}^{2}}(l=1, \cdots, m)$ as local coordinates. If we choose a number $a$ such that $0<a<1$ $-\frac{2}{m}+\min \varepsilon_{i}$, then we have

$$
\begin{aligned}
& (a-1) \sum_{i, j=1}^{m} p_{i j} \frac{\partial y_{m}}{\partial x_{i}} \frac{\partial y_{m}}{\partial x_{j}} y_{m}^{a-2}+\sum_{i, j=1}^{m} \frac{\partial}{\partial x_{j}}\left(p_{i j} \frac{\partial y_{m}}{\partial x_{i}}\right) y_{m}^{a-1} \\
& =(a-1) \sum_{k=1}^{m}\left(1-\sqrt{\sum_{k=1}^{m} x_{k}^{2}}\right)^{2 / m-\varepsilon_{i}}\left(\frac{\partial y_{m}}{\partial x_{i}}\right)^{2} y_{m}^{a-2} \\
& +\sum_{i=1}^{m} \frac{\partial}{\partial x_{i}}\left\{\left(1-\sqrt{\sum_{k=1}^{m} x_{k}^{2}}\right)^{2 / m-\varepsilon_{i}} \frac{\partial y_{m}}{\partial x_{i}}\right\} y_{m}^{a-1} \\
& \approx(a-1) \sum_{i=1}^{m}\left(1-\sqrt{\sum_{k=1}^{m} x_{k}^{2}}\right)^{2 / m-\varepsilon_{i}}\left(\frac{x_{i}}{\sqrt{\sum_{k=1}^{m} x_{k}^{2}}}\right)^{2} y_{m}^{a-2} \\
& +\sum_{i=1}^{m}\left(\frac{2}{m}-\varepsilon_{i}\right)\left(1-\sqrt{\sum_{k=1}^{m} x_{k}^{2}}\right)^{2 / m-\varepsilon_{i}-1}\left(\frac{x_{i}}{\sqrt{\sum_{k=1}^{m} x_{k}^{2}}}\right)^{2} y_{m}^{a-1} \\
& =\sum_{i=1}^{m}\left\{(a-1)+\left(\frac{2}{m}-\varepsilon_{i}\right)\right\}\left(\frac{x_{i}}{\sqrt{\sum_{k=1}^{m} x_{k}^{2}}}\right)^{2} y_{m}^{2 / m-\varepsilon_{i}+a-1} \\
& \leqq \sum_{i=1}^{m}\left\{(a-1)+\left(\frac{2}{m}-\min \varepsilon_{i}\right)\right\}\left(\frac{x_{i}}{\sqrt{\sum_{k=1}^{m} x_{k}^{2}}}\right)^{2} y_{m}^{a-1+2 / m-\max \varepsilon_{i}} \text {. }
\end{aligned}
$$




$$
=\left\{(a-1)+\left(\frac{2}{m}-\min \varepsilon_{i}\right)\right\} y_{m}^{a-1+2 \mid m-\max \varepsilon_{i}} .
$$

Thus the inequality (6) is satisfied.

2) Let

$$
\Omega=\left\{x \mid 0<x_{i}<1(i=1, \cdots, m)(m \geqq 2)\right\} .
$$

Consider the problem

$$
\begin{aligned}
& \sum_{i=1}^{m} \frac{\partial}{\partial x_{i}}\left(x_{i}^{2 / m-\varepsilon_{i}} \frac{\partial u}{\partial x_{i}}\right)-q u=f\left(0<\varepsilon_{i} \leqq \frac{2}{m}\right) \text { in } \Omega, \\
& u=\varphi \quad \text { on } \partial \Omega,
\end{aligned}
$$

where

$$
\begin{aligned}
& q, f \in C_{0, \alpha}(\Omega) \cap C_{0,0}(\bar{\Omega}), \\
& \varphi \in C_{2, \alpha}(\Omega) \cap C_{0,0}(\bar{\Omega})
\end{aligned}
$$

and

$$
q>0 \text { in } \bar{\Omega}(q \neq 0 \text { at } x=0) .
$$

Again this problem has a unique solution.

3) Let

$$
\Omega=\left\{x \mid 1<\sum_{k=1}^{m} x_{k}^{2}<4(m \geqq 2)\right\} .
$$

Consider the eigenvalue problem for

$$
\begin{aligned}
& \sum_{i=1}^{m \prime} \frac{\partial}{\partial x_{i}}\left\{\left(\sqrt{\sum_{k=1}^{m} x_{k}^{2}}-1\right)^{2 / m-\varepsilon_{i}} \frac{\partial u}{\partial x_{i}}\right\} \\
& +\sum_{i=m^{\prime}+1}^{m} \frac{\partial}{\partial x_{i}}\left\{\left(2-\sqrt{\sum_{k=1}^{m} x_{k}^{2}}\right)^{2 / m-\varepsilon_{i}} \frac{\partial u}{\partial x_{i}}\right\}-q u+\lambda \rho u=0 \\
& \left(0<\varepsilon_{i} \leqq \frac{2}{m}, 1 \leqq m^{\prime}<m\right) \text { in } \Omega \text {, } \\
& u=0 \quad \text { on } \partial \Omega \text {, }
\end{aligned}
$$

where

$$
q, \rho, f \in C_{0, \alpha}(\Omega) \cap C_{0,0}(\bar{\Omega}),
$$

and

$$
\rho \geqq \rho_{0}>0 \quad \text { in } \bar{\Omega} .
$$

This problem has countable and discrete eigenvalues. 
4) Let

$$
\Omega=\left\{x \mid 0<x_{i}<1(i=1, \cdots, 2 m)(m \geqq 1)\right\} .
$$

Consider the eigenvalue problem for

$$
\begin{gathered}
\sum_{i=1}^{m} \frac{\partial}{\partial x_{i}}\left(x_{i}^{1 / m-\varepsilon_{i}} \frac{\partial u}{\partial x_{i}}\right)+\sum_{i=1}^{m} \frac{\partial}{\partial x_{m+i}}\left\{\left(1-x_{i}\right)^{1 / m-\varepsilon_{i}} \frac{\partial u}{\partial x_{m+i}}\right\} \\
-q u+\lambda \rho u=0\left(0<\varepsilon_{i} \leqq \frac{1}{m}\right) \text { in } \Omega, \\
u=0 \quad \text { on } \partial \Omega,
\end{gathered}
$$

where

$$
q, \rho, f \in C_{0^{\prime} \alpha}(\Omega) \cap C_{0,0}(\bar{\Omega})
$$

and

$$
\rho \geqq \rho_{0}>0 \text { in } \bar{\Omega}
$$

Again this problem has countable and discrete eigenvalues.

\section{REFERENCES}

[1] Courant, R. and D. Hilbert, Methods of mathematical physics, II, Interscience, New York, 1953.

[2] I'l in, A.M., Degenerate elliptic and parabolic equations, Mat. Sb. 50 (1960), 443-498. (Russian)

[3] Ladyzhenskaya, O.A. and N. N. Ural'tseva, Linear and quasi-linear equations of elliptic type, Izdatel'stvo Nauka, Moskva, 1964. (Russian)

[4] Mikhlin, S. G., Linear equations in mathematical physics, Izdatel'stvo Nauka, Moskva, 1964. (Russian)

[5] Nirenberg, L., On elliptic partial differential equations, Ann. Scuola Norm. Sup. Pisa, 13 (1959), 115-162.

[6] Oleinik, O.A., On the linear equations of the second order with non-negative characteristic form, Mat. Sb. 69 (1966), 111-140. (Russian)

[7] Schauder, J., Über lineare elliptische Differentialgleichungen zweiter Ordnung, Math. Z. 38 (1934), 257-282.

[8] Smirnov, V. I., A course to higher mathematics, V, Gosudarstvennoe Isdatel'stvo Fiziko-Matematicheskoi Literatury, Moskva, 1959. (Russian)

[9] Suzuki, K., On eigenvalue problems (1), The Memoirs of Gakushuin High School, No.2 (1966), 26-76. (Japanese) 
